NIST

PUBLICATIONS

NISTIR 3969

\title{
A SURVEY OF
}

CURRENT WORLDWIDE RESEARCH ON

THE THERMOPHYSICAL PROPERTIES

OF ALTERNATIVE REFRIGERANTS

M. O. McLinden

W. M. Haynes

J. T. R. Watson

K. Watanabe

QC

100

1056

3969

1991

C.2 



\section{NISTIR 3969}

\section{A SURVEY OF \\ CURRENT WORLDWIDE RESEARCH ON THE THERMOPHYSICAL PROPERTIES OF ALTERNATIVE REFRIGERANTS}

M. O. McLinden

W. M. Haynes

J. T. R. Watson*

K. Watanabe ${ }^{\star *}$

Thermophysics Division

Chemical Science and Technology Laboratory

National Institute of Standards and Technology

Boulder, Colorado 80303-3328

*Energy and Environment Centre

National Engineering Laboratory

Glasgow, United Kingdom

${ }^{\star \star}$ Department of Mechanical Engineering

Keio University

Yokohama, Japan

June 1991

Sponsored by

U.S. Department of Energy

Office of Buildings and Community Systems

1000 Independence Ave., SW

Washington, DC 20585

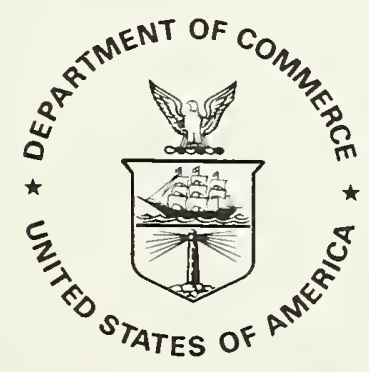

U.S. DEPARTMENT OF COMMERCE, Robert A. Mosbacher, Secretary NATIONAL INSTITUTE OF STANDARDS AND TECHNOLOGY, John W. Lyons, Director 


\section{ABSTRACT}

This survey represents an exhaustive compilation of the research activities throughout the world concerned with either measurements or correlations of the thermophysical properties of alternative refrigerants. The properties covered in this study include thermodynamic, transport, phase equilibria, and other properties such as dielectric constant and refractive index. This survey has included a wide range of fluids (including R23, R32, R125, R143a, R22, R134a, R152a, R134, R124, R142b, R123, R123a, R141b), along with mixtures containing at least one of these fluids. This report presents in tabular form summary information about each research activity; this survey does not present raw data or correlating equations.

Key words: hydrochlorofluorocarbons; hydrofluorocarbons; refrigerants; survey; thermodynamic properties; thermophysical properties; transport properties 
INTRODUCTION

Table 1-Summary of Alternative Refrigerants

SURVEY PROCEDURE

Figure 1-Survey Form

SUMMARY OF SURVEY RESULTS .

Table 2 - Summary of Research by Fluid and Property . . . . . . . . . . . . 6

Table 3 - Summary of Research by Fluid and Country . . . . . . . . . . . . . 7

Table 4 -Summary of Mixtures Research . . . . . . . . . . . . . . . . . . 8

ACKNOWLEDGMENTS $\ldots \ldots \ldots \ldots \ldots \ldots \ldots \ldots \ldots \ldots \ldots \ldots \ldots$

DETAILED PROJECT INFORMATION $\ldots \ldots \ldots \ldots \ldots \ldots \ldots \ldots \ldots$

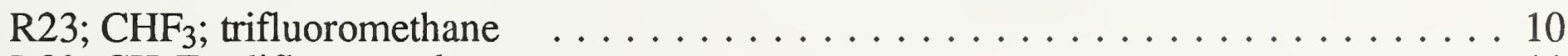

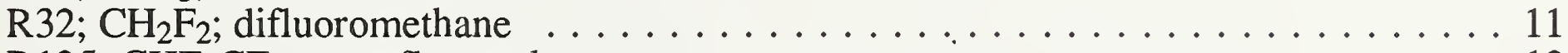

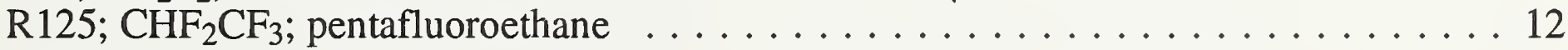

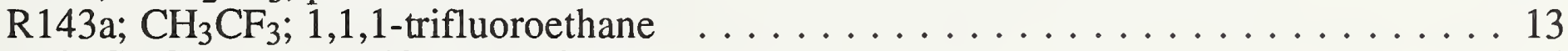

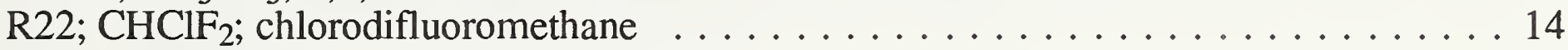

R134a; $\mathrm{CH}_{2} \mathrm{FCF}_{3} ; 1,1,1,2$-tetrafluoroethane . . . . . . . . . . . . . . 17

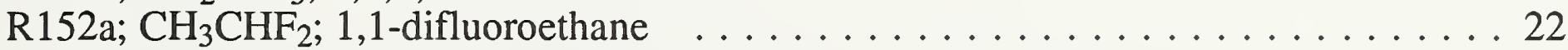

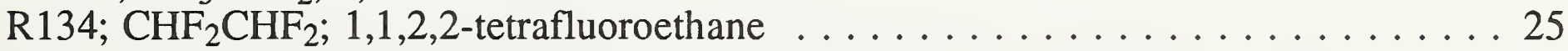

R124; $\mathrm{CHClFCF}_{3}$; 1-chloro-1,2,2,2-tetrafluoroethane . . . . . . . . . . . 27

$\mathrm{R} 142 \mathrm{~b} ; \mathrm{CClF}_{2} \mathrm{CH}_{3} ; 1$-chloro-1,1-difluoroethane . . . . . . . . . . . 28

$\mathrm{R} 123 ; \mathrm{CHCl}_{2} \mathrm{CF}_{3} ; 1$ 1,-dichloro-2,2,2-trifluoroethane $\ldots \ldots \ldots \ldots \ldots \ldots \ldots \ldots$

$\mathrm{R} 123 \mathrm{a} ; \mathrm{CHClFCClF}_{2} ; 1,2$-dichloro-1,2,2-trifluoroethane $\ldots \ldots \ldots \ldots \ldots \ldots$

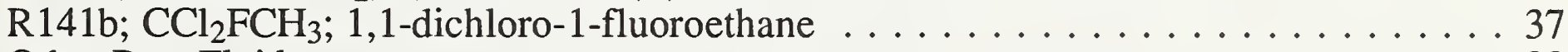

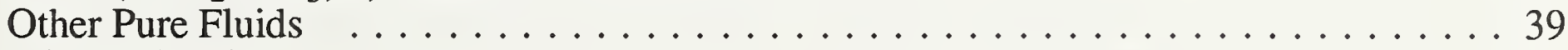

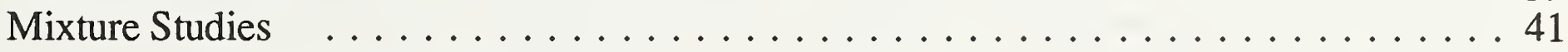

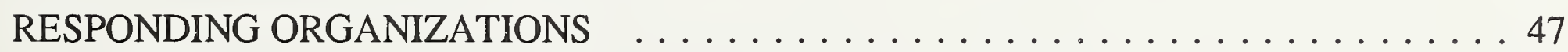

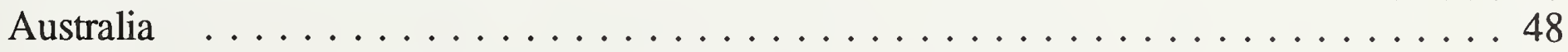

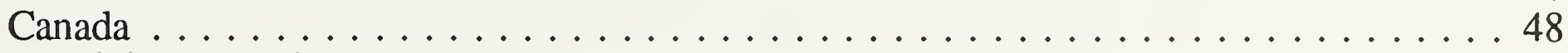

People's Republic of China . . . . . . . . . . . . . . . . . . . . . 50

Czechoslovakia ............................ 52

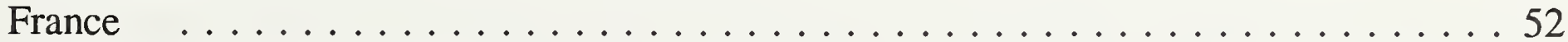

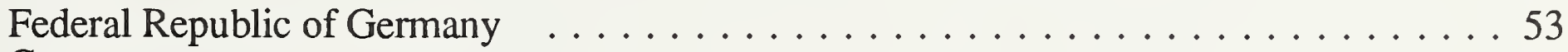

Greece ................................... 60

India . . . . . . . . . . . . . . . . . . . . . . 60

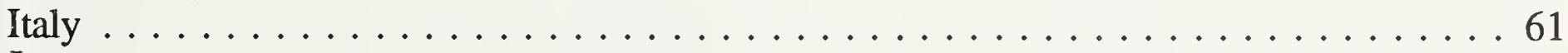

Japan . . . . ............................662

Korea ................................... 67

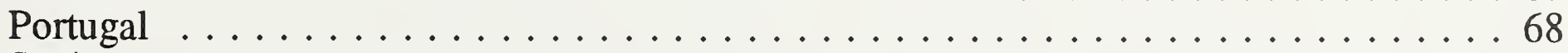

Spain ................................ 68

Sweden . . . . . . . . . . . . . . . . . . . . . . . . . . . . . . 69

Union of Soviet Socialist Republics . . . . . . . . . . . . . . . . . . . . . . 69

United Kingdom . . . . . . . . . . . . . . . . . . . . . . . 70

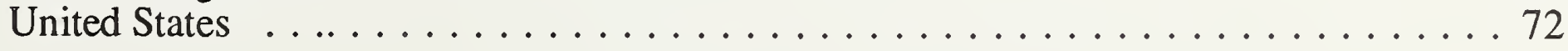





\section{INTRODUCTION}

The advent of the Montreal Protocol banning the future production of the fully halogenated chlorofluorocarbon (CFC) refrigerants, which are major contributors to the environmental problems of ozone depletion and greenhouse warming, has presented a major challenge to the refrigeration industry. The expected prospect of also phasing out hydrochlorofluorocarbons (HCFCs) sometime early in the next century adds to this challenge. There are a number of very promising fluids under study which may serve as substitutes, either as pure fluids, as constituents of mixtures, or both. These substitutions must not, however, be done at the expense of energy efficiency. In order to evaluate the performance (energy efficiency, capacity, etc.) of these fluids in any thermodynamic cycle, a knowledge of their thermophysical properties is required. Accurate values of these properties are essential to select, from a set of closely related fluids or fluid mixtures, the working fluid that will yield the highest energy efficiency in refrigeration and heatpumping applications.

The results of a comprehensive survey of current worldwide research on the thermophysical properties of alternative refrigerants are reported here in tabular form. This work summarizes under a single cover an exhaustive compilation of these activities. Its objectives are (1) to assist in the coordination of existing research programs so as to expedite the acquisition of the required property data and to not unnecessarily duplicate research programs and (2) to serve as a guide for planning the future directions of research projects involving the thermophysical properties of alternative refrigerants.

The traditional approach to determining the thermophysical properties of a given fluid is an exacting, time-consuming, and specialized endeavor. In order to expedite the effort to achieve an accurate knowledge of the thermophysical properties of environmentally acceptable refrigerants, it was appropriate to establish an international collaborative effort to provide a forum for the exchange of information and data and for the coordination of activities. Toward this end, the Advanced Heat Pump Programme of the International Energy Agency established Annex 18: "Thermophysical Properties of the Environmentally Acceptable Refrigerants." The ultimate goal of this Annex is to provide property formulations that will become de facto international standards for the most promising candidates. The first step necessary to accomplish this goal is to identify the sources of experimental data and correlations. Therefore, this report of current research activities on the thermophysical properties of alternative refrigerants represents the first major task of Annex 18. This report presents in tabular form the results of this task; this survey will be updated as deemed necessary by the development of new research activities in this area.

This survey represents an exhaustive compilation of the research activities throughout the world concerned with either measurements or correlations of the thermophysical properties of alternative refrigerants. The properties covered in this study include thermodynamic, transport, phase equilibria, and other properties such as dielectric constant and refractive index. This survey has covered a wide range of fluids (including R23, R32, R125, R143a, R22, R134a, R152a, $\mathrm{R} 134, \mathrm{R} 124, \mathrm{R} 142 \mathrm{~b}, \mathrm{R} 123, \mathrm{R} 123 \mathrm{a}, \mathrm{R} 141 \mathrm{~b}$ ), along with mixtures containing at least one of these fluids. These fluids are listed with their molecular mass, normal boiling point, etc. in Table 1. This report presents in tabular form summary information about each research activity; this survey does not present raw data or correlating equations. Future IEA collaborative tasks include the measurement, compilation, and evaluation of experimental data for the most important alternatives, along with the development and recommendation of correlations. 
Table 1-Summary of Alternative Refrigerants

$\begin{array}{llrrr}\begin{array}{l}\text { Fluid } \\ \text { number }\end{array} & \text { formula } & \begin{array}{c}\mathrm{MW} \\ (\mathrm{g} / \mathrm{mol})\end{array} & \begin{array}{c}\mathrm{NBP} \\ \left({ }^{\circ} \mathrm{C}\right)\end{array} & \begin{array}{c}\mathrm{T}_{\mathrm{c}} \\ \left({ }^{\circ} \mathrm{C}\right)\end{array} \\ \text { R23 } & \mathrm{CHF}_{3} & 70.01 & -82.1 & 25.9 \\ \text { R32 } & \mathrm{CH}_{2} \mathrm{~F}_{2} & 52.02 & -51.8 & 78.4 \\ \text { R125 } & \mathrm{CF}_{3} \mathrm{CHF}_{2} & 120.02 & -48.6 & 66.3 \\ \text { R143a } & \mathrm{CF}_{3} \mathrm{CH}_{3} & 84.04 & -47.4 & 73.1 \\ \text { R22 } & \mathrm{CHClF}_{2} & 86.47 & -40.8 & 96.2 \\ \text { R134a } & \mathrm{CF}_{3} \mathrm{CH}_{2} \mathrm{~F} & 102.03 & -26.1 & 101.1 \\ \text { R152a } & \mathrm{CHF}_{2} \mathrm{CH}_{3} & 66.05 & -24.2 & 113.3 \\ \text { R134 } & \mathrm{CHF}_{2} \mathrm{CHF}_{2} & 102.03 & -19.4 & 119.0 \\ \text { R124 } & \mathrm{CHClFCF}_{3} & 136.48 & -12.1 & 122.5 \\ \text { R142b } & \mathrm{CClF}_{2} \mathrm{CH}_{3} & 100.50 & -9.3 & 137.1 \\ \text { R123 } & \mathrm{CHCl}_{2} \mathrm{CF}_{3} & 152.93 & 27.9 & 183.8 \\ \text { R123a } & \mathrm{CHClFCClF}_{2} & 152.93 & 29.8 & 185.8 \\ \text { R141b } & \mathrm{CCl}_{2} \mathrm{FCH}_{3} & 116.95 & 32.0 & 204.7\end{array}$


The survey was conducted by Annex 18 participants from Japan, the United Kingdom, and the United States, with the U.S. participants acting as overall coordinators. A comprehensive survey form (Figure 1) was developed and in the early summer of 1990 was sent to over 120 research groups worldwide that were either known to be active in refrigerant research or that have fluid property expertise. Responses were received from more than 60 research groups in 15 countries. Additional responses were received but were not included in the summary presented here because the work fell outside the scope of the survey (e.g., heat transfer measurements on alternative refrigerants) or was proprietary and insufficient information was provided. Studies of refrigerant-oil solubility were not included here even though they were on the survey form; the very limited number of responses received were not felt to be representative of the extensive work in this area either because we did not query the appropriate research groups or because refrigerantoil research is largely proprietary.

The focus of the survey is on current studies of refrigerant thermophysical properties. Also solicited in the survey and included here are recent work (i.e., work completed since 1988) and future work. Although the survey instructions specified that future work be included only if a definite commitment for funding had been established, many of the projects listed several years in the future, or simply as "planned," probably do not meet this criterion. References for published results during the past two years are also reported.

This report has been distributed to all participants in IEA Annex 18 as well as to all survey respondents. This will hopefully facilitate the objectives of this Annex. 
Project type: $\square$ experimental measurement $\square$ modeling/correlation

Fluid(s) studied:

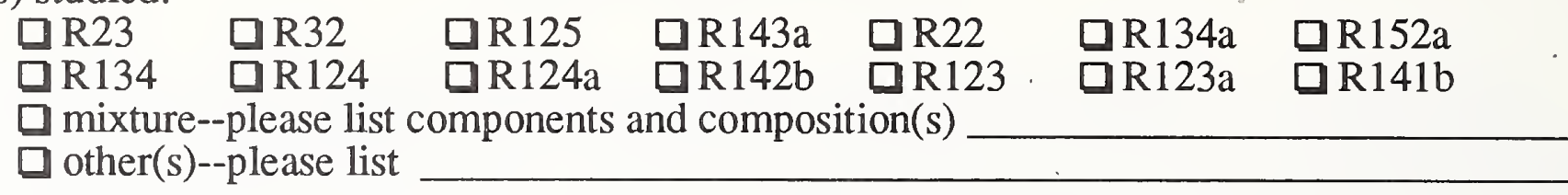
sample purity
$\square$ by own analysis
by suppliers analysis
estimated/unknown

Property(ies) measured/correlated (also indicate number of experimental points):
$\square$ vapor pressure (
$\square$ P-V-T
$\square$ saturation density $(\stackrel{)}{(})$
$\square$ critical parameters ( $\square$ dielectric constant $(\square)$
$\square$ heat capacity (
$\square$ surface tension
$\square$ sound speed
$\square$ viscosity (
$\square$ thermal conductivity (
$\square$ refractive index
$\square$ refrigerant/oil solubility (_
$\square$ other(s)--please list

Experimental technique/correlating equation employed:

Estimated accuracy (experimental projects only)

Range of measurements/correlation (either indicate numerically or sketch below)
temperature: from
pressure: from
to
density:
from
to
phase:
$\square$ saturated liquid
to
a single-phase liquid
$\square$ saturated vapor
$\square$ critical region
$\square$ single-phase vapor
$\square$ supercritical region

Single-phase properties

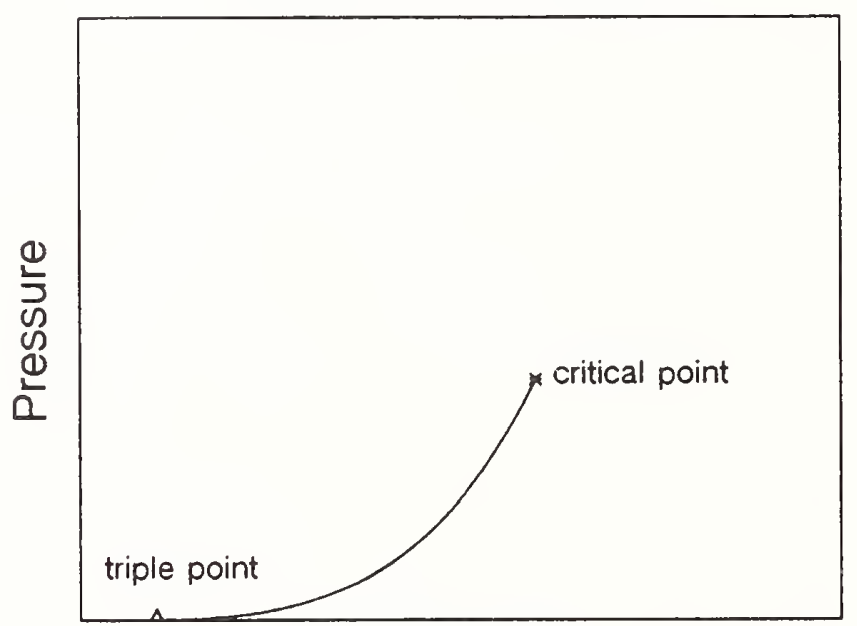

Temperature
Saturation properties

property

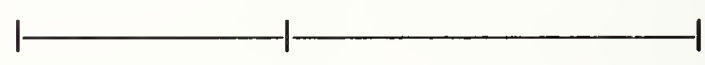

property

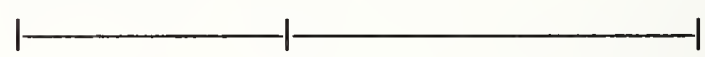

property

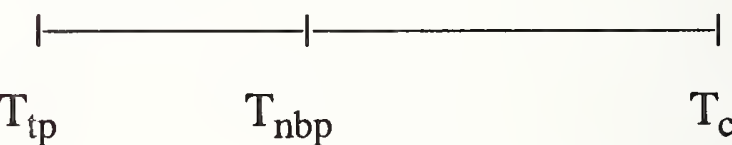

Project status:

$\square$ completed--if paper or report is available please give reference:

$\square$ in progress

$\square$ planned (i.e. funded) beginning date

ending date

Additional remarks: 
A series of tables on the following pages presents the results of the survey in a compact format. Tables 2,3 , and 4 summarize the number of projects by fluid, type of property measured, and the country in which the work is being done. These tables tally the "number of projects" for a particular combination of fluid and country, or fluid and property. Each "project" represents one property studied for one fluid by one research group. A research program involving the measurement of a single property for several fluids, several different property measurements for one fluid, or a project being carried out by more than one laboratory would be counted multiple times in computing the entries in Tables $2-4$. Similarly, a project measuring only a few data points is counted the same as an exhaustive set of measurements. Thus, the total quantity of work on the alternative refrigerants is considerably less than what might initially be inferred from Tables 2-4. These tables do, however, give an indication of the countries active in studying refrigerant thermophysical properties and the relative level of effort being expended for the various alternatives.

Following the summary tables, a series of tables, one for each fluid, present details of the responses. The fluids are listed in order of increasing normal boiling point; mixtures follow the pure components and are listed first in order of the normal boiling point of the more volatile component, and then by the normal boiling point of the other constituent(s). For a given fluid, the entries are further organized by property. The information presented for each entry are selfevident. Dashes indicate missing information or an item that is not applicable. The order of the entries reflects the sequence of their entry into the tables and has no other significance. Each entry is identified by the name of the person responding to the survey (usually the principal investigator), the organization, and the country. The final set of tables, organized by country, gives details on the research groups responding to this survey. Names, addresses, and telephone numbers of the principal investigator(s) and any other collaborators are given, as well as a brief listing of the techniques employed. The listing of techniques represents those that have been applied to the alternative refrigerants; in many cases these research groups have additional capabilities.

\section{ACKNOWLEDGMENTS}

We extend our sincere thanks to all of our colleagues from around the world who shared with us information on their work on the alternative refrigerants. The U.S. participation in this task was funded by the Office of Buildings and Community Systems of the United States Department of Energy. 


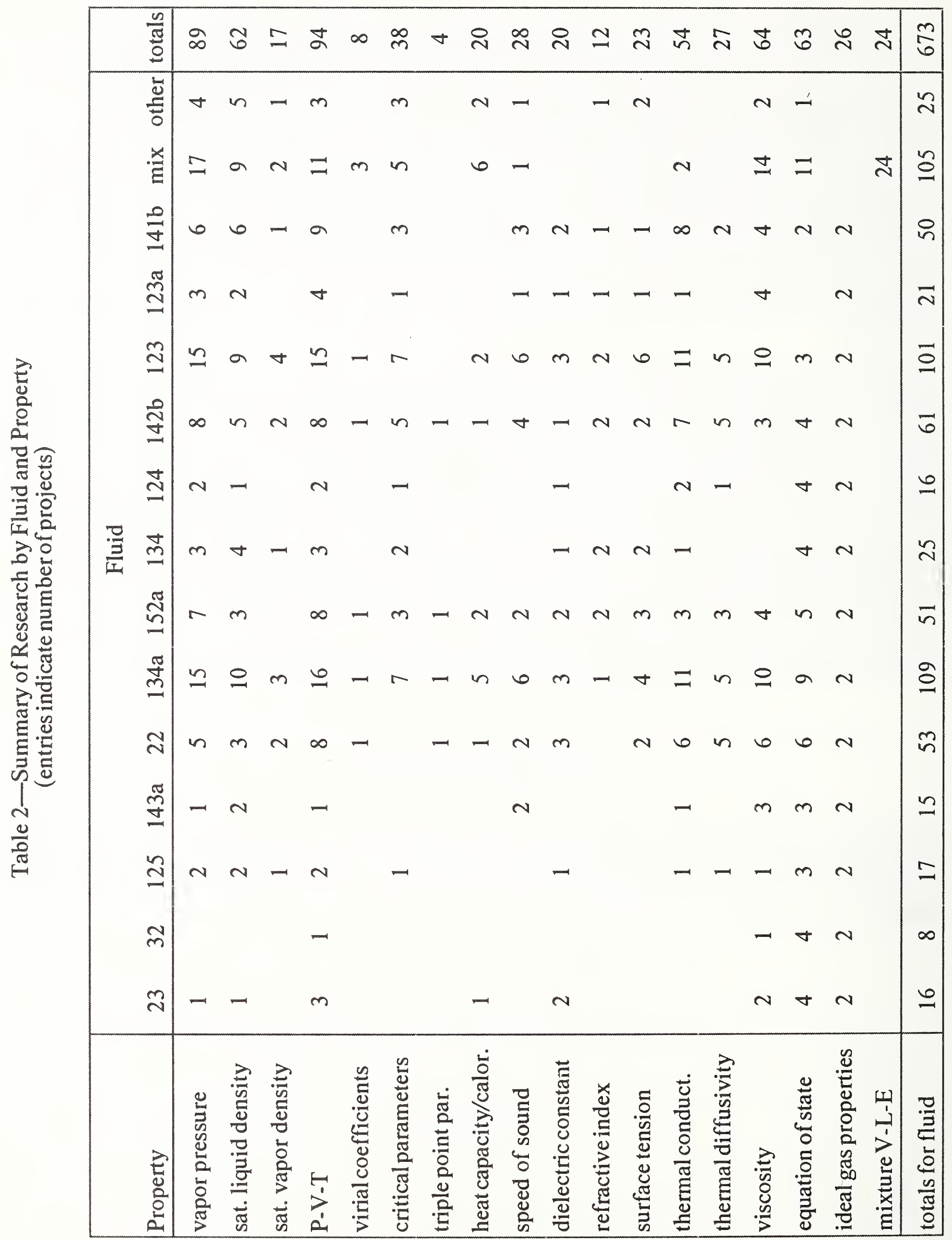




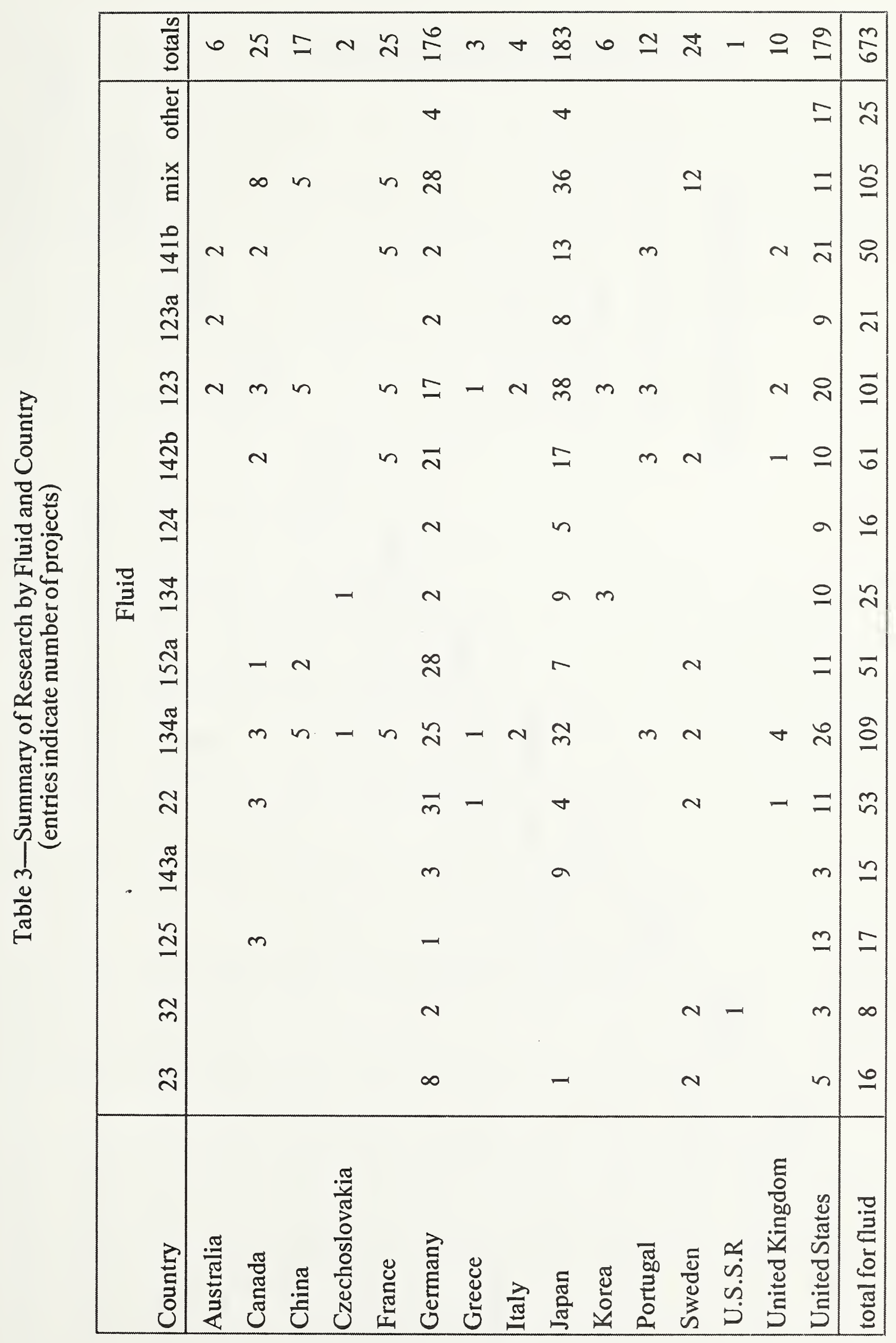




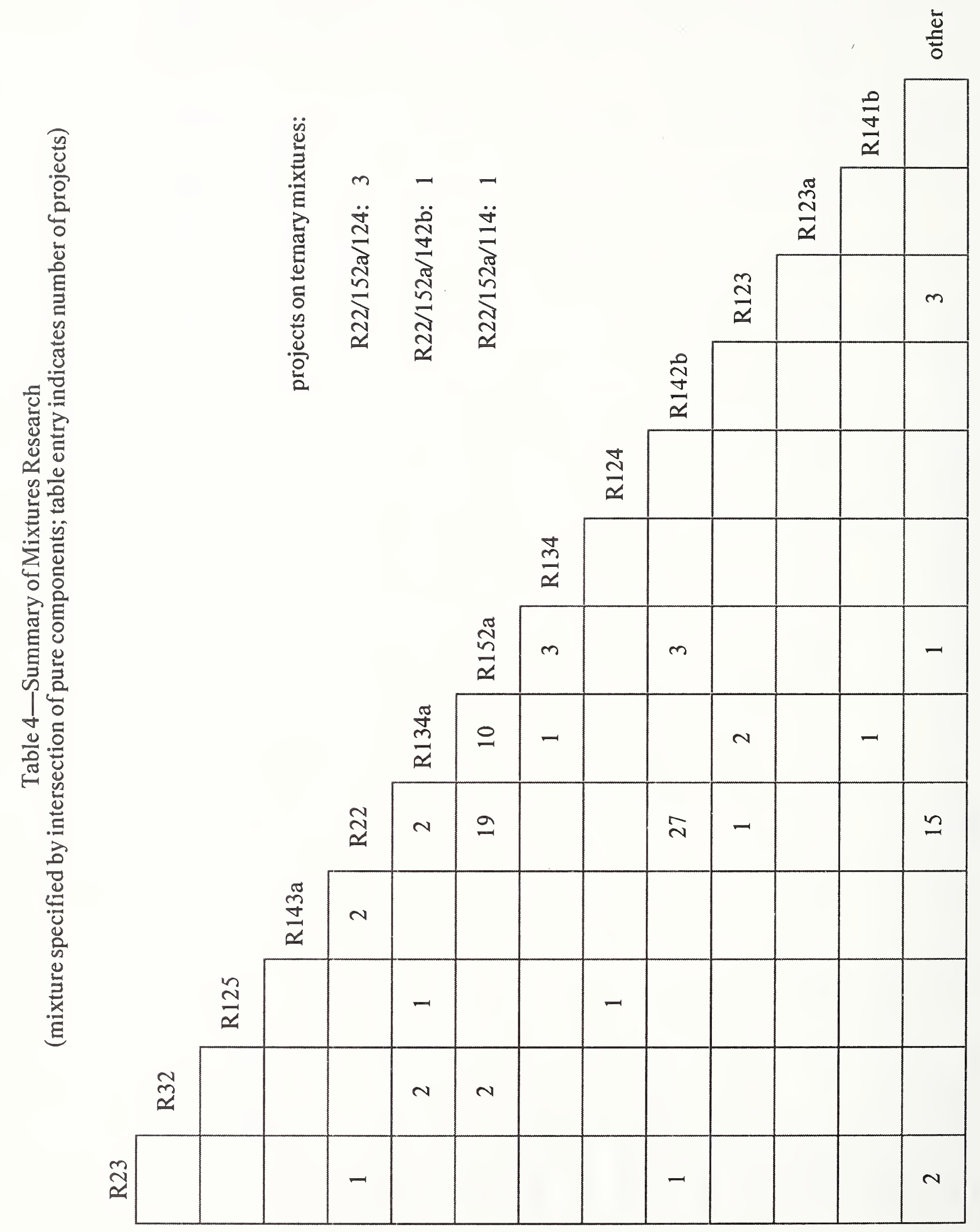


DETAILED PROJECT INFORMATION 
Property

/Investigator

Vapor pressure

Kruse; U. Hannover; FRG

Saturated liquid density

Kruse; U. Hannover; FRG

Pressure-volume-temperature

Ström; Chalmers U. Tech.; Swe

Zollweg; Cornell U.; USA

Bier; U. Karlsrühe; FRG

Heat capacity/calorimetry

Bier; U. Karlsrühe; FRG

Dielectric constant

Makita; Kobe U.; Japan

Franck; U. Karlsruhe; FRG

$V$ iscosity

Ström; Chalmers U. Tech.; Swe

Kruse; U. Hannover; FRG

Equation of State

Maurer; U. Kaiserlautern; FRG

Huber; NIST; USA

Fischer; ORNL; USA

Gallagher; NIST; USA phase

range of data

$\mathrm{T}(\mathrm{K})$

$\mathrm{p}(\mathrm{MPa})$

no.

est.

sample

purity

start/end

dates

note

/ref.

$1-$

-v $\quad-$

sl

$--$

l 258-323

$0.5-2$

-- $\quad 0.15 \%$

sat-100

$1300 \quad 0.1-0.4 \%$

$128 \quad 0.02-0.14 \%$

.99

.999

.999

planned

comp

comp

[Rb89]

v $333-423$

$0.2-58$

$0.1-12$

163

$0.2 \%$

.999

comp

l,v 283-323

0.1-17

126

$0.01 \%$

.9991

comp

l,v 213-468

0.5-200

$90 \quad 0.5-1.5 \%$

.9995

comp

[Rt89]

$\begin{array}{ccccc}1 & 258-323 & 0.5-2 & -- & 2 \% \\ \mathrm{~s} 1, \mathrm{sv} & -- & - & -- & --\end{array}$

.99

planned

9/89-8/91

comp

[P190]

in prog

[E190]

comp

[Fs90]

$\mathrm{l}, \mathrm{v}$

l,v $0.5-1.6 \mathrm{~T}_{\mathrm{c}}$

$0-2 \mathrm{P}_{\mathrm{c}}$

comp

[G190]

Ideal Gas Properties

Lucas; U. Duisburg; FRG

- $100-500$

0-0

$1 \%$

in prog

comp

[TR90]

Notes and References

[E190] J. F. Ely and M. L. Huber, Proc. ASHRAE-Purdue CFC Conference, W. Lafayette, IN, USA, July, 1990, pp 383-392.

[Fs90] S. K. Fischer and J. Sand, Proc. ASHRAE-Purdue CFC Conference, W. Lafayette, IN, USA, July, 1990, pp 373-382.

[G190] J. Gallagher, M. McLinden, and G. Morrison, National Institute of Standards and Technology, Standard Reference Data Base 23 (1990).

[P190] B. Platzer, A. W. Polt, and G. Maurer, Thermophysical Properties of Refrigerants, Springer-Verlag, Heidelberg (1990).

[Rb89] R. G. Rubio, J. A. Zollweg, and W. B. Streett, Ber. Bunsenges. Phys. Chem. 93791 (1989).

[Rt89] K. Reuter, S. Rosenzweig, and E.U. Franck, The static dielectric constant of $\mathrm{CH}_{3} \mathrm{~F}$ and $\mathrm{CHF}_{3}$ to $468 \mathrm{~K}$ and 2000 bar, Physica A 156 294-302 (1989).

[TR90] TRC Thermoaynamic Tables, Thermodynamics Research Center, Texas A\&M University, continuously updated; also to be published in J. Phys. Chem. Ref. Data. 
$\mathrm{R} 32 ; \mathrm{CH}_{2} \mathrm{~F}_{2}$; difluoromethane

Property

/Investigator

phase range of data no. est.

$\mathrm{T}(\mathrm{K}) \quad \mathrm{p}(\mathrm{MPa})$ points accuracy purity dates

note

Pressure-volume-temperature

Ström; Chalmers U. Tech.; Swe

$1 \quad 258-323$

$0.5-2 \quad--\quad 0.15 \%$

.99 planned

Viscosity

Ström; Chalmers U. Tech.; Swe

$1 \quad 258-323$

$0.5-2$

$2 \%$

.99 planned

Ideal Gas Properties

Lucas; U. Duisburg; FRG

- $100-500$

$0-0$

$1 \%$

in prog

- $0-1500$

0.1

$--$

comp

[TR90]

Equation of State

Los; Inst. Low Temp. Engr.; USSR

$\begin{array}{cc}\mathrm{v} & 200-500 \\ \mathrm{l,v} & 190-400 \\ \mathrm{l}, \mathrm{v} & \text { all } \\ \mathrm{l,v} & -\end{array}$

$0-5$

$0-6$

Morgenstern; HFV Dresden; FRG

Huber; NIST; USA

Fischer; ORNL; USA

all

all

Notes and References

[E190] J. F. Ely and M. L. Huber, Proc. ASHRAE-Purdue CFC Conference, W. Lafayette, IN, USA, July, 1990, pp 383-392.

[Fs90] S. K. Fischer and J. Sand, Proc. ASHRAE-Purdue CFC Conference, W. Lafayette, IN, USA, July, 1990, pp 373-382.

[TR90] TRC Thermodynamic Tables, Thermodynamics Research Center, Texas A\&M University, continuously updated; also to be published in J. Phys. Chem. Ref. Data. 
$\mathrm{R} 125$; $\mathrm{CHF}_{2} \mathrm{CF}_{3}$; pentafluoroethane

Property

/Investigator

Vapor pressure

Holste; Texas A\&M U.; USA

Morrison; NIST; USA

Saturated liquid density

Holste; Texas A\&M U.; USA

Morrison; NIST; USA

Saturated vapor density

Holste; Texas A\&M U.; USA

Pressure-volume-temperature

Holste; Texas A\&M U.; USA

Morrison; NIST; USA

Critical parameters

Holste; Texas A\&M U.; USA

Dielectric constant

Morrison; NIST; USA

Thermal conductivity

Venart; U. New Brunswick; Canada

$1, v \quad 203-393$

$0.1-70$

$-$

$0.1 \%$

sl 268-339

sv $\quad 230-480$

$$
1, \mathrm{~V} \quad 230-480
$$

0-70

sat -7

60

$0.1 \%$

$0.1 \%$

$\mathrm{T}, \mathrm{P}$,

v 298-433

$0.04-1.4$

$3 \times 10^{-6}$

.993

comp

[My90]

$--$

.993

9/90-10/91

comp

9/90-10/91

9/90-10/91

.993 comp

$9 / 90-10 / 91$

$0.1 \%$

$-$

(a)

planned

Thernal diffusivity

Venart; U. New Brunswick; Canada

$1, v \quad 203-393$

$0.1-70$

$5 \%$

planned

Viscosity

Snelson; NRC; Canada

sl 253-353

$15 \quad 2.5-3.5 \%$

3/91-10/91

Equation of state

Huber; NIST; USA

Fischer; ORNL; USA

Gallagher; NIST; USA

Ideal Gas Properties

Lucas; U. Duisburg; FRG

Marsh; Texas A\&M; USA

\begin{tabular}{|c|c|}
\hline $1, \mathrm{v}$ & all \\
\hline $1, v$ & - \\
\hline $0.5-1.6 \mathrm{~T}_{\mathrm{c}}$ & \\
\hline
\end{tabular}

- $100-500$

0-0

0.1
$1 \%$

$--$ in prog

comp

comp

[E190]

[Fs90]
[G190]

Notes and References

[E190] J. F. Ely and M. L. Huber, Proc. ASHRAE-Purdue CFC Conference, W. Lafayette, IN, USA, July, 1990, pp 383-392.

[Fs90] S. K. Fischer and J. Sand, Proc. ASHRAE-Purdue CFC Conference, W. Lafayette, IN, USA, July, 1990, pp 373-382.

[G190] J. Gallagher, M. McLinden, and G. Morrison, National Institute of Standards and Technology, Standard Reference Data Base 23 (1990).

[My90] C. W. Meyer and G. Morrison, J. Phys. Chem. (in press).

[TR90] TRC Thermodynamic Tables, Thermodynamics Research Center, Texas A\&M University, continuously updated; also to be published in J. Phys. Chem. Ref. Data. 
Property

Investigator

Vapor pressure

Widiatmo; Keio U.; Japan

Saturated liquid density

C. Yokoyama; Tohoku U.; Japan

Widiatmo; Keio U.; Japan

Pressure-volume-temperature

Widiatmo; Keio U.; Japan

Speed of sound

Kohler; Ruhr U.;FRG

Takagi; Kyoto Inst. Tech.; Japan

Thermal conductivity

Tanaka; Kobe U.; Japan

Viscosity

Takahashi; Tohoku U.; Japan

Kumagai; Tohoku U.; Japan

Tanaka; Kobe U.; Japan

Equation of State phase $\mathrm{T}(\mathrm{K})$

range of data

no.

est.

$\mathrm{p}(\mathrm{MPa})$ points accuracy l-v 200-345

s! $\quad 250-340$

sl 200-345

$1 \quad 280-315$

sat-2

$-$

$0.2 \%$

$--$

$7 / 90-3 / 91$

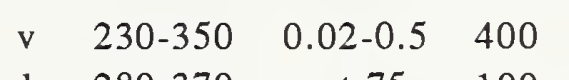

$1 \quad 280-370 \quad$ sat $-75 \quad 100$

$0.01 \%$

$0.2 \%$

.993

.9996

$? / 88-? / 91$

comp comp

7/90-3/91

v 293-353

0.1-sat 30

$2 \%$

.999

comp

[Tn90]

$\begin{array}{cccccccc}\mathrm{v} & 298-423 & 0.1-9 & 107 & 0.3 \% & .999 & \text { comp } & \text { [Tk90] } \\ \mathrm{s} 1 & 273-323 & \text { sat } & 6 & 0.5 \% & .999 & \text { comp } & \text { [Km90] } \\ \mathrm{v} & 283-323 & 0.1-0.1 & 5 & 1 \% & -- & \text { comp } & \text { [Ko89] }\end{array}$

Morgenstern; HFV Dresden; FRG

Huber; NIST; USA

Fischer; ORNL; USA
$1, \mathrm{v} \quad 200-400$
$\mathrm{l}, \mathrm{v}$ all
$1, \mathrm{v} \quad-$

$0-4$

all

$-$

$-$

$1 \%$

$0-0$

$-\quad 100-500$
$-\quad 0-1500$

0.1

comp
in prog
comp

[E190]

[Fs90]

Ideal Gas Properties

Lucas; U. Duisburg; FRG

Marsh; Texas A\&M; USA

Notes and References

[E190] J. F. Ely and M. L. Huber, Proc. ASHRAE-Purdue CFC Conference, W. Lafayette, IN, USA, July, 1990, pp 383-392.

[Fs90] S. K. Fischer and J. Sand, Proc. ASHRAE-Purdue CFC Conference, W. Lafayette, IN, USA, July, 1990, pp 373-382.

[Km90] A. Kumagai, to be published in Int. J. Thermophysics (1990).

[Ko89] thesis, Kobe University (1989).

[Tk90] M. Takahashi, to be presented at 31st High Pressure Conference of Japan, November, 1990, Osaka.

[Tn90] Y. Tanaka, submitted to Int. J. Thermophysics (1990).

[TR90] TRC Thermodynamic Tables, Thermodynamics Research Center, Texas A\&M University, continuously updated; also to be published in J. Phys. Chem. Ref. Data. 
R22; $\mathrm{CHClF}_{2}$; chlorodifluoromethane

Property

Investigator phase

range of data
$\mathrm{T}(\mathrm{K})$

p (MPa) no.

est.

sample

start/end

note

Vapor pressure

Blanke; PTB; FRG

Gorenflo; U. Paderborn; FRG

Kohler; Ruhr U.; FRG

Straub; T.U. München; FRG

Wagner; Ruhr U.; FRG

Saturated liquid density

Gorenflo; U. Paderborn; FRG

Wagner; Ruhr U.; FRG

Kruse; U. Hannover; FRG

Saturated vapor density

Gorenflo; U. Paderborn; FRG

Wagner; Ruhr U.; FRG

$\begin{array}{lr}\text { SV } & 293-366 \\ \text { SV } & 250-330\end{array}$

$\begin{array}{ccc}- & 16 & 0.2 \% \\ - & 4 & 0.02 \%\end{array}$

.9999

.99995

comp

[R+89]

comp [Wg90]

Pressure-volume-temperature

Blanke; PTB; FRG

Speed of sound

Dielectric constant

Surface Tension

Kohler; Ruhr U.; FRG

Straub; T.U. München; FRG

Ström; Chalmers U. Tech.; Swe

Wagner; Ruhr U.; FRG

Kubota; Kobe U.; Japan

Morrison; NIST; USA

Sandarusi; NIST; USA

$\begin{array}{cccc}\text { sat-30 } & 300 & 0.1 \% & .9999 \\ 0.1-60 & 280 & 0.05 \% & .9996 \\ 2-12 & 300 & 0.5-1.5 \% & .9998 \\ 0.5-2 & -- & 0.15 \% & .99 \\ 0-8 & 55 & 0.02 \% & .99995 \\ 0.1-11 & 56 & 0.4 \% & .999 \\ \text { sat-7 } & 60 & 0.1 \% & -- \\ 1.4-40 & 400 & 0.1-0.3 \% & .999+\end{array}$

l,v $\quad 318-473$

l $113-470$

$1, \mathrm{v} \quad 250-500$

$1, \mathrm{v} \quad 318-413$

l 258-323

$1, \mathrm{v} \quad 120-340$

v $348-423$

l 268-378

$1.4-40$ v 233-296

9

$2 \%$

comp

[Nt89]

Schramm; Phys. Chem. Inst.; FRG

\footnotetext{
(
}

Triple point temperature

Blanke; PTB; FRG

Heat capacity/calorimetry

Sami; U. Moncton; Canada

l,v 283-353

$0.5-1.4$

$2 \%$

.9999

$10 / 86-9 / 90$

Kohler; Ruhr U.; FRG

v 230-350

$0.02-0.5 \quad 400$

$0.01 \%$

.9996

$? / 88-? / 91$

Leipertz; U. Erlangen-Nürnberg; FRG

$\mathrm{l}, \mathrm{v} \quad 240-450$

$0.5 \%$

--

4/90-->
Makita; Kobe U.; Japan

Franck; U. Karlsruhe; FRG

Morrison; NIST; USA

$$
\begin{array}{cr}
\mathrm{v} & 293-373 \\
\mathrm{l}, \mathrm{v} & 233-368 \\
\mathrm{v} & 298-433
\end{array}
$$

\section{.9999}

.994

$0.5-200 \quad 90$

$0.7 \%$

$3 \times 10^{-6}$ comp

comp

comp

[Um89]

[My90]
Straub; T.U. München; FRG

I-v 223-369

100

$0.5 \%$

.9998

$36 \quad 0.16 \mathrm{mN} / \mathrm{m}$ planned comp
[Kh90]

[Wg90]

Okada; Nagaoka U. Tech.; Japan 
R22; $\mathrm{CHClF}_{2}$; chlorodifluoromethane (continued)

Property

Investigator phase

$$
\mathrm{T}(\mathrm{K})
$$

range of data

$\mathrm{p}(\mathrm{MPa})$ no.

points accuracy sample

purity start/end dates

note

Thermal conductivity

$\begin{array}{cc}-- & 6 / 89-? \\ .9999 & \text { comp } \quad \text { [Hm89] } \\ .99996 & -->9 / 91 \\ -- & 12 / 90-12 / 92 \\ -- & 1 / 91-9 / 92 \\ -- & \text { in prog }\end{array}$

Assael; Aristotle U.; Greece

Hemminger; PTB; FRG

Stephan/Taxis; U. Stuggart; FRG

Johns; Nat'l. Eng. Lab; UK

Perkins; NIST; USA

Venart; U. New Brunswick; Canada

$\begin{array}{ccccc}1, \mathrm{v} & 230-370 & <40 & - & 0.5 \% \\ \mathrm{v} & 303-463 & 0.1-0.1 & 5 & 2.5 \% \\ \mathrm{v} & 273-423 & <5 & 100 & 1 \% \\ \mathrm{v} & 298-423 & 1-30 & -- & 0.5 \% \\ 1, \mathrm{v} & 130-405 & 0.1-70 & 800 & 1 \% \\ 1, \mathrm{v} & 203-393 & 0.1-70 & -- & .1 \%\end{array}$

$1 \quad 273-363$

$1, v \quad 240-450$

$1, \mathrm{v} \quad 318-413$

$1, \mathrm{v} \quad 130-405$

$1, v \quad 203-393$

$$
1-8
$$

$<15$

2-12

$0.1-70$

$0.1-70$

$\begin{array}{cc}-- & 1 \% \\ -- & 1-2 \% \\ 300 & 0.5-1.5 \% \\ 800 & 5 \% \\ -- & 5 \%\end{array}$

Venart; U. New Brunswick; Canada

Fiebig; Ruhr U.; FRG

Leipertz; U. Erlangen-Nürnberg; FRG

Straub; T.U. München; FRG

Perkins; NIST; USA

iscosity

Mayinger; T. U. München; FRG

Ström; Chalmers U. Tech.; Swe

Kumagai; Tohoku U.; Japan

Kruse; U. Hannover; FRG

Diller; NIST; USA

Diller; NIST; USA v $300-425$

l 258-323

sl 273-323

l 236-353

$1 \quad 120-320$

$\mathrm{l}, \mathrm{v} \quad 300-500$

$\begin{array}{cc}.995 & \text { comp } \\ -- & 4 / 90--> \\ .9998 & \text { comp } \\ -- & 1 / 91-9 / 92 \\ -- & \text { in prog }\end{array}$

$\begin{array}{ccc}0.1-7.5 & 35 & 0.5 \% \\ 0.5-2 & -- & 2 \% \\ \text { sat } & 6 & 0.5 \% \\ ?-2.5 & 8 & 4 \% \\ \text { sat-35 } & 60 & 3 \% \\ 0.2-50 & 100 & 3 \%\end{array}$

.9995

comp planned

.99

.999 .999

comp

comp

comp

10/90-10/91

$\begin{array}{lcc}\mathrm{l}, \mathrm{v} & 253-473 & 0-35 \\ \mathrm{l}, \mathrm{v} & 115-525 & 0-160 \\ \mathrm{l,v} & - & - \\ \mathrm{l,v} & \text { all } & \text { all } \\ \mathrm{l}, \mathrm{v} & - & - \\ \mathrm{l}, \mathrm{v} & 0.5-1.6 \mathrm{~T}_{\mathrm{c}} & 0-2 \mathrm{P}_{\mathrm{c}} \\ \mathrm{l,v} & - & -\end{array}$

- $100-500$

$0-0$

$1 \%$

- $0-1500$

0.1

$--$

comp
$-->3 / 91$
$? / 89-? / 92$

in prog

[E190]

comp [Fs90]

comp [G190]

comp

in prog

[P190]

comp

[TR90] 


\section{$\mathrm{R} 22 ; \mathrm{CHClF}_{2}$; chlorodifluoromethane (continued)}

Notes and References

[E190] J. F. Ely and M. L. Huber, Proc. ASHRAE-Purdue CFC Conference, W. Lafayette, IN, USA, July, 1990, pp 383-392.

[Fs90] S. K. Fischer and J. Sand, Proc. ASHRAE-Purdue CFC Conference, W. Lafayette, IN, USA, July, 1990, pp 373-382.

[G190] J. Gallagher, M. McLinden, and G. Morrison, National Institute of Standards and Technology, Standard Reference Data Base 23 (1990).

[Hm89] U. Hammerschmidt and W. Hemminger, DKV-Tagungsberict 16 397-410 (1989).

[Kh90] R. Kohlen, Fortschrittberichte VDI, Reihe 19, No. 14 (19??).

[Km90] A. Kumagai, to be published in Int. J. Thermophysics (1990).

[My90] C. W. Meyer and G. Morrison, J. Phys. Chem. (in press).

[Nb90] H. Nabizadeh and F. Mayinger, Viscosity of refrigerants R22, R114 and mixture of R22+R114 at superheated and supercritical states, paper presented at AIChE Spring National Meeting, Orlando, Florida, March, 1990.

[Nt89] G. Natour, H. Schuhmacher, and B. Schramm, Second virial coefficients and intermolecular forces in the system $\mathrm{CCl}_{2} \mathrm{~F}_{2} / \mathrm{CHClF}_{2}$, Fluid Phase Equilibria 49 67-74 (1989).

[Ok88] M. Okada, J. Chem. Eng. Data 33 399-401 (1988).

[P190] B. Platzer, A. W. Polt, and G. Maurer, Thermophysical Properties of Refrigerants, Springer-Verlag, Heidelberg (1990).

[Rt89] W. Rott, dissertation, Universität -GH- Paderborn (1989).

[TR90] TRC Thermodynamic Tables, Thermodynamics Research Center, Texas A\&M University, continuously updated; also to be published in J. Phys. Chem. Ref. Data.

[Um89] M. Uematsu and E. U. Franck, The dielectric constant of chlorodifluoromethane to $200 \mathrm{MPa}$, Ber. Bunsenges. Phys. Chem. 93 177-180 (1989).

[Wg90] W. Wagner, to be submitted to J. Chem. Thermodyn. (1990). 
R134a; $\mathrm{CH}_{2} \mathrm{FCF}_{3} ; 1,1,1,2$-tetrafluoroethane

Property

Investigator phase range of data no. est. $\mathrm{T}(\mathrm{K}) \quad \mathrm{p}(\mathrm{MPa})$ points accuracy sample

start/end dates /ref.

Vapor pressure

Baehr; U. Hannover; FRG

Baroncini; U. Ancona; Italy

LeNeindre; U. Paris Nord; France

Straub; T.U. München; FRG

Oguchi; Kanagawa Inst. Tech.; Japan

Kubota; Kobe U.; Japan

Fukushima; Asahi Glass; Japan

Maezawa; Keio U.; Japan

Piao; Keio U.; Japan

Mingshan; Tsinghua U.; PRC

Kruse; U. Hannover; FRG

Howley; NIST; USA

Bier; U. Karlsrühe; FRG

Weber; NIST; USA

Morrison; NIST; USA

$\begin{array}{lc}l-v & 300-374 \\ 1-v & 240-360 \\ 1-v & 298-? \\ 1-v & 318-374 \\ 1-v & 243-374 \\ 1-v & 253-373 \\ l-v & 262-374 \\ 1-v & 280-350 \\ l-v & 300-374 \\ l-v & 248-358 \\ 1-v & -- \\ l-v & 180-360 \\ l-v & 203-374 \\ l-v & 313-374 \\ l-v & 268-374\end{array}$

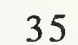

64

0.03
$1 \%$

$--$

37

\section{5}

41

13

51

20

$$
--
$$

19

53

22

10 note /ref.

Saturated liquid density

Baehr; U. Hannover; FRG

sl 293-373

9

$0.1 \%$

$>.9993$

8/89-8/91

s1 252-367

22

$0.5 \%$

.999

comp

[Yk88]

Oguchi; Kanagawa Inst. Tech.; Japan

s1 243-346

Fukushima; Asahi Glass; Japan

sl 244-374

$6 \quad 0.01-0.1 \%$

.999

comp

$16 \quad 0.2-0.3 \%$

.9999

sl $200-370$

25

$0.2 \%$

.9999

Piao; Keio U.; Japan

sl $313-372$

$0.15 \%$

$.995-.9999$

comp

comp

[Mz90]

$11 \quad 0.5 \%$

.995

comp

[Pi90]

s1 354-374

Kruse; U. Hannover; FRG

Holste; Texas A\&M U.; USA

Morrison; NIST; USA

s1

s1 $180-360$

$--$

--

comp

$--$

$0.1 \%$

.9997

.9994

9/89-8/91

comp

comp

[Ka89]

10

-

sv $\quad 365-374$

sv $271-374$

sv $320-365$

Weber; NIST; USA

Kabata; Keio U.; Japan

$\begin{array}{ccccc}- & 11 & 0.5 \% & .995 & \text { comp } \\ - & 8 & 3 \mathrm{~kg} / \mathrm{m}^{3} & .9999 & \text { comp } \\ - & 5 & 0.02 \% & .9995 & \text { comp }\end{array}$

[Ka89]

[Wb89] 


\section{R134a; $\mathrm{CH}_{2} \mathrm{FCF}_{3} ; 1,1,1,2$-tetrafluoroethane (continued)}

Property

Investigator phase

range of data $\mathrm{T}(\mathrm{K})$
$\mathrm{p}(\mathrm{MPa})$ points accuracy sample

start/end

note

purity dates /ref.

Pressure-volume-temperature

Baehr; U. Hannover; FRG

Baroncini; U. Ancona; Italy

LeNeindre; U. Paris; France

Nieto de Castro; U. Lisbon; Por.

Straub; T.U. München; FRG

Ström; Chalmers U.Tech.; Swe.

Vacek; Czech T.U.; Czech.

Oguchi; Kanagawa Inst. Tech.; Japan

Matsuo; Kobe U.; Japan

Fukushima; Asahi Glass; Japan

Maezawa; Keio U.; Japan

Piao; Keio U.; Japan

Mingshan; Tsinghua U.; PRC

Holste; Texas A\&M U.; USA

Weber; NIST; USA

Morrison; NIST; USA

$\begin{array}{cc}\mathrm{l}, \mathrm{v} & 293-433 \\ \mathrm{v}, \mathrm{sv} & 240-360 \\ \mathrm{l}, \mathrm{v} & 298-413 \\ \mathrm{l}, \mathrm{v} & <\mathrm{T}_{\mathrm{c}} \\ \mathrm{l}, \mathrm{v} & 318-423 \\ \mathrm{l} & 258-323 \\ \mathrm{l} & 200-300 \\ \mathrm{l} & 243-473 \\ \mathrm{l} & 293-353 \\ \mathrm{l}, \mathrm{v} & 320-424 \\ \mathrm{l} & 280-350 \\ \mathrm{l}, \mathrm{v} & 300-425 \\ \mathrm{v} & 248-358 \\ \mathrm{l} & 180-380 \\ \mathrm{v} & 321-423 \\ \mathrm{l} & 268-378\end{array}$

$\begin{array}{cc}0.1-16 & 500 \\ 0.1-\text { sat } & 46 \\ 0.1-100 & -- \\ <20 & -- \\ 2-12 & 300 \\ 0.5-2 & 120 \\ \text { sat-55 } & -- \\ 0.08-17 & 40 \\ \text { sat-40 } & 50 \\ 1-6 & 63 \\ \text { sat-2 } & 16 \\ 0.7-12 & 159 \\ 0.1-6 & 84 \\ \text { sat-70 } & 300 \\ 0-5.3 & 69 \\ \text { sat-7 } & 60\end{array}$

$0.1 \%$

$1 \%$

$--$

$0.3 \%$

$0.5-1.5 \%$

$0.15 \%$

$0.1 \%$

$0.01 \%$

$0.02 \%$

$0.2 \%$

$0.2 \%$

$0.15 \%$

$0.2 \%$

$0.1 \%$

$0.02 \%$

$0.1 \%$

9

Virial coefficients

Schramm; Phys.-Chem. Inst.; FRG

v 233-296

Critical parameters

LeNeindre; U. Paris; France

Straub; T.U. München; FRG

Fukushima; Asahi Glass; Japan

Piao; Keio U.; Japan

T, $\rho$

Kabata; Keio U.; Japan

T,p

Kubota; Kobe U.; Japan

$\mathrm{T}, \mathrm{P}$

Bier; U. Karlsrühe; FRG

Triple point temperature

Magee; NIST; USA

Heat capacity/calorimetry

Wormald; U. Bristol; UK

Piao; Keio U.; Japan

Saitoh; Keio U.; Japan

Magee; NIST; USA

Magee; NIST; USA

$\begin{array}{cc}\mathrm{l}, \mathrm{v} & 233-473 \\ -- & -- \\ \mathrm{l} & 276-356 \\ \mathrm{l} & 190-340 \\ \mathrm{l}, \mathrm{v} & 320-500\end{array}$

$0.1-10$

$-$

$1-3$

sat -35

0.1-20

Speed of sound

Kohler; Ruhr U.; FRG

Leipertz; U. Erlangen-Nürnberg; FRG

LeNeindre; U. Paris; France

Mingshan; Tsinghua U.; PRC

Zollweg; Cornell U.; USA

Goodwin; NIST; USA

$\begin{array}{cccc}\mathrm{v} & 230-350 & .025-.5 & 400 \\ \mathrm{l,v} & 240-450 & <15 & -- \\ \mathrm{l,v} & 298-523 & 0.1-100 & -- \\ \mathrm{v} & 248-358 & 0.1-1.5 & -- \\ \mathrm{l} & 180-380 & \text { sat-70 } & 206 \\ \mathrm{v} & 233-340 & 0.02-.5 * \text { Psat } 104\end{array}$

$0.01 \%$

$0.5 \%$

$--$

$0.15 \%$

$0.05 \%$

$0.01 \%$
$.9993+$ .9998

$--$

.995

.999

.99

--

.999

.999

.9999

.9999

$.995-.9999$

.9998

.9997

.9995

.9994

$1 \%$
--
$0.3 \%$
$0.5 \%$
$0.4 \%$

.

8/89-8/91

comp [Br90]

10/90-->

12/89-10/93

$1 / 89-8 / 90$

$6 / 91-12 / 91$

comp

comp

comp

comp

comp

comp

comp [H190]

comp [Wb89]

comp

[Mz90]

[Pi90]

[Wb89]

6/90-->

$10 / 90->$

$1 / 89-8 / 90$

$5 \mathrm{mK}, 3 \mathrm{~kg} / \mathrm{m}^{3} \quad .9999 \quad$ comp

$2 \mathrm{kPa} \quad .995-.9999$ comp

[Pi90]

comp [Ka89]

comp

[Kb89]

comp

[Bi90]

.999

.9995

comp

[Mg91]

$\begin{array}{ccr}.99+ & 4 / 90-12 / 91 & \\ .995-.9999 & \text { comp } & {[\mathrm{Pi} 90]} \\ .9997 & \text { comp } & {[\mathrm{St90}]} \\ .9995 & \text { comp } & {[\mathrm{Mg} 91]} \\ .9997 & 10 / 91-12 / 91 & \end{array}$

-- ?/88-?/91

-- 4/90-->

-- 10/90-->

$.9998 \quad 4 / 90-->$

$.998 \quad$ comp

[Z190]

.9994 comp

[Gd90] 
Property

Investigator phase range of data no. est. $\mathrm{T}(\mathrm{K}) \quad \mathrm{p}(\mathrm{MPa})$ points accuracy sample purity start/end note dates /ref.

Dielectric constant

Nieto de Castro; U. Lisbon; Por.

Makita; Kobe U.; Japan

Morrison; NIST; USA $\begin{array}{cc}\mathrm{l}, \mathrm{v} & <\mathrm{Tc} \\ \mathrm{v} & 298-348 \\ \mathrm{v} & 298-433\end{array}$

$<20$

$0.04-0.7$

29

$0.5 \%$

$0.5 \%$

$3 \times 10^{-6}$
$1, v \quad 318-423$

Straub; T.U. München; FRG

Surface Tension

Okada; Nagaoka U. Tech.; Japan

Mingshan; Tsinghua U.; PRC

Straub; T.U. München; FRG

Schmidt; NIST; USA l-v 263-358

l-v 223-373

l-v 263-368 l-v 232-363
300

$0.05 \%$

$\begin{array}{cc}118 & 0.2 \mathrm{mN} / \mathrm{m} \\ -- & -- \\ 100 & 0.5 \% \\ 29 & 0.15 \mathrm{mN} / \mathrm{m}\end{array}$
.995

.999

.9994

12/89-10/93

comp

comp [My90]

$.999 \quad 1 / 89-8 / 90$

$\begin{array}{cc}.9998 & \text { comp } \\ .9998 & 4 / 90--> \\ .998 & \text { planned } \\ .9995 & \text { comp }\end{array}$

[Ok90]

comp

[Ch90]

Thermal conductivity

Assael; Aristotle U.; Greece

Gross/Hahne; U. Stuggart; FRG

LeNeindre; U. Paris Nord; France

Nieto de Castro; U. Lisbon; Por.

Stephan/Taxis; U. Stuggart; FRG

Wakeham; Imperial Col.; UK

Yata; Kyoto Inst. Tech.; Japan

Tanaka; Kobe U.; Japan

Nagasaka; Keio U.; Japan

Perkins; NIST; USA

Venart; U. New Brunswick; Canada

$\begin{array}{cccc}\mathrm{l}, \mathrm{v} & 230-370 & <40 & -- \\ \mathrm{l}, \mathrm{v} & 262-354 & 0.1-8.0 & 71 \\ \mathrm{l}, \mathrm{v} & 298-523 & 0.1-100 & -- \\ \mathrm{l}, \mathrm{v} & <\mathrm{T}_{\mathrm{c}} & <20 & -- \\ \mathrm{v} & 273-423 & <5 & 100 \\ \mathrm{sl,sv} & 260-370 & \text { sat } & 20 \\ \mathrm{l} & 250-350 & \text { sat-30 } & 127 \\ \mathrm{v} & 293-353 & 0.1-\mathrm{sat} & 33 \\ \mathrm{l} & 193-273 & 1-1 & -- \\ \mathrm{l,v} & 180-410 & 0.1-70 & 800 \\ \mathrm{l}, \mathrm{v} & 203-393 & 0.1-70 & --\end{array}$

$0.5 \%$

$1 \%$

$--$

$0.5 \%$

$1 \%$

--

--

$2 \%$

$0.5 \%$

$1 \%$

$1 \%$
[Gr90]

$10 / 90-->$

2/89-10/93

-->9/91

comp

[Wk90]

[Yt89]

[Tn90]

?/89-->

-->12/90

planned
1-8

$<15$

2-12

$0.1-70$

$0.1-70$
-- $1 \%$

$-$

300

800

l,v 203-393
$1-2 \%$

$0.5-1.5 \%$

$5 \%$

$5 \%$

$\begin{array}{cc}-- & 1 / 91--> \\ -- & 4 / 90--> \\ .999 & 12 / 89-8 / 90 \\ -- & -->12 / 90 \\ -- & \text { planned }\end{array}$

-.

.9994

.99

.999

.999

.9998

--

$\begin{array}{ll}-- & 9 / 89-8 / 91 \\ -- & \text { comp }\end{array}$

-- $10 / 90-10 / 91$

3/91-10/91

$-$

3/91-10/91

$60 \quad 3 \%$

100

15
$3 \%$

$2.5-3.5 \%$
1/90-->

comp

[My90]

comp

[Tk.90]

comp

4/90-->

9/89-8/91

[Km90]

Snelson; NRC; Canada

l 175-320

l,v $\quad 300-500$

sl 253-353

$\begin{array}{cc}\text { v } & 247-573 \\ \text { v } & 300-425 \\ \text { l } & 258-323 \\ \text { v } & 298-423 \\ \text { sl } & 273-343 \\ \text { sl } & 263-358 \\ \text { l,sv } & -- \\ \text { l } & 175-320 \\ \text { l,v } & 300-500 \\ \text { sl } & 253-353\end{array}$

$-$ 
Property

Investigator

Equation of State

Piao; Keio U.; Japan

Maurer; U. Kaiserlautern; FRG

Wakeham; Imperial Col., UK

Sengers; U. Maryland; USA

Ely; NIST; USA

Oellrich; U. Karlsrühe; FRG

Huber; NIST; USA

Fischer; ORNL; USA

Gallagher; NIST; USA phase range of data $\mathrm{T}(\mathrm{K})$ no. points accuracy sample purity start/end dates note

/ref.

Ideal Gas Properties

Lucas; U. Duisburg; FRG

Marsh; Texas A\&M; USA

$\begin{array}{lcc}\mathrm{l}, \mathrm{v} & 300-425 & 0-12 \\ \mathrm{l}, \mathrm{v} & 210-500 & 0-12 \\ \mathrm{l}, \mathrm{v} & -- & -- \\ \mathrm{l,v} & 356-450 & 3-12 \\ \mathrm{l,v} & 170-450 & 0-70 \\ \mathrm{l,v} & - & - \\ \mathrm{l}, \mathrm{v} & \text { all } & \text { all } \\ \mathrm{l,v} & - & - \\ \mathrm{l,v} & 0.5-1.6 \mathrm{~T}_{\mathrm{c}} & 0-2 \mathrm{P}_{\mathrm{c}}\end{array}$

$\begin{array}{ll}- & - \\ - & - \\ - & - \\ - & - \\ - & - \\ - & - \\ - & - \\ - & - \\ - & -\end{array}$

-
-
-
-
-
-
-
-
-

\begin{tabular}{|c|c|}
\hline comp & [Pi90] \\
\hline comp & [P189] \\
\hline planned & \\
\hline comp & [Sn90] \\
\hline $3 / 90-12 / 90$ & [E190] \\
\hline ?/89-?/92 & \\
\hline in prog & \\
\hline comp & \\
\hline comp & \\
\hline
\end{tabular}

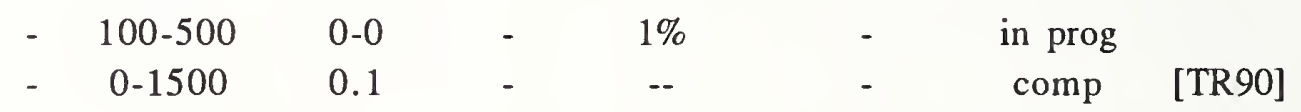

Notes and References

[Bi90] K. Bier, R. Hamberger, M. Weisenburger, and J. Zhai; DKV-Jahrestagung 22/23 (1990).

[Br90] C. Baroncini, to be published in Proc., IIR Commission B1 Meeting, Tel-Aviv, March, 1990.

[Ch90] H. B. Chae, J. W. Schmidt, and M. R. Moldover, J. Chem. Eng Data 356.

[E190] J. F. Ely, to be submitted to Int. J. Refrigeration (1990).

[E190a] J. F. Ely and M. L. Huber, Proc. ASHRAE-Purdue CFC Conference, W. Lafayette, IN, USA, July, 1990, pp 383-392.

[Fs90] S. K. Fischer and J. Sand, Proc. ASHRAE-Purdue CFC Conference, W. Lafayette, IN, USA, July, 1990, pp 373-382.

[Gd90] A. R. H. Goodwin and M. R. Moldover, J. Chem. Phys 93 2741-.

[G190] J. Gallagher, M. McLinden, and G. Morrison, National Institute of Standards and Technology, Standard Reference Data Base 23 (1990).

[Gr90] U. Gross and E. Hahne, to be published in Proc., IIR Commission B1 Meeting, Tel-Aviv, March, 1990.

[H190] J. C. Holste, to be submitted to Int. J. Refrigeration (1990).

[Hw90] J. Howley, to be submitted to Int. J. Refrigeration (1990).

[Ka89] Y. Kabata, S. Tanikawa, M. Uematsu, and K. Watanabe; Int. J. Thermophysics 10 605-615 (1989).

[Kb89] H. Kubota, Int. J. Thermophysics 10629 (1989)

[Km90] A. Kumagai, to be published in Int. J. Thermophysics.

[Mo90] G. Morrison and D. K. Ward, Fluid Phase Equilibrium (in press).

[My90] C. W. Meyer and G. Morrison, J. Phys. Chem. (in press).

[Mz90] Y. Maezawa, J. Chem. Eng. Data, 35 (1990), in press.

[Mg91] J. W. Magee, to be submitted to Int. J. Refrigeration (1991).

[My90] F. Mayinger and H. Nabizadeh, data to be published September, 1990.

[Ok90] M. Okada, to be published in Proc., IIR Commission B1 Meeting, Tel-Aviv, March, 1990.

[Pi90] C.-C. Piao, H. Sato, and K. Watanabe, ASHRAE Trans. 96 [part 1] (1990).

[P189] B. Platzer and G. Maurer, ESCAG: Ein Programmpaket zur Berechnung thermodynamischer Zustandsgrößen reiner Fluide mit Hilfe der Zustandsgleichung von Bender, 16th DKV-Tagungsbericht, Bd. 2, 441-456 (1989).

[St90] A. Saitoh, S. Nakagawa, H. Sato, and K. Watanabe; J. Chem. Eng. Data 35107 (1990).

[Sn90] J. V. Sengers and S. Tang, paper in preparation (1990).

[Tk90] M. Takahashi, Proc., 30th High Pressure Conference of Japan, p. 372 (1990).

[Tn90] Y. Tanaka, submitted to Int. J. Thermophysics. 
R134a; $\mathrm{CH}_{2} \mathrm{FCF}_{3} ; 1,1,1$,2-tetrafluoroethane (continued)

Notes and References (continued)

[TR90] TRC Thermodynamic Tables, Thermodynamics Research Center, Texas A\&M University, continuously updated; also to be published in J. Phys. Chem. Ref. Data.

[Wb89] L. A. Weber, Int. J. Thermophysics 10 617-627.

[Wk90] W. Wakeham, data presented in proprietary report.

[Yt89] J. Yata, et al., Thermal conductivity of R123 and R134a in liquid phase, Proc. 2nd Asian Thermophysical Properties Conference, p. 201 (1989).

[Yk88] C. Yokoyama, Proc., 29th High Pressure Conference of Japan, p. 116.

[Z190] J. A. Zollweg; to be submitted to Int. J. Refrigeration (1990). 
Property

Investigator phase $\mathrm{T}(\mathrm{K})$ $\begin{array}{ccc}\text { of data } & \text { no. } & \text { est. } \\ p(\mathrm{MPa}) & \text { points } & \text { accuracy }\end{array}$ sample purity start/end dates

note /ref.

Vapor pressure

Baehr; U. Hannover; FRG

Blanke; PTB; FRG

Straub; T.U. München; FRG

Yin; Xi'an Jiaotong U.; PRC

Kruse; U. Hannover; FRG

Bier; U. Karlsrühe; FRG

Morrison; NIST; USA

$$
\begin{array}{cc}
l-v & 300-386 \\
l-v & 156-386 \\
l-v & 330-386 \\
l-v & 242-293 \\
1-v & -- \\
l-v & 203-386 \\
l-v & 268-383
\end{array}
$$

Saturated liquid density

Baehr; U. Hannover; FRG

$\begin{array}{ll}\text { s1 } & 293-383 \\ \text { si } & 233-353 \\ \text { sl } & 268-383\end{array}$

-
-

$0.1 \%$

$0.3 \%$

Morrison; NIST; USA

Pressure-volume-temperature

Baehr; U. Hannover; FRG
Blanke; PTB; FRG
Straub; T.U. München; FRG
Ström; Chalmers U. Tech.; Swe
Kuböta; Kobe U.; Japan
Kubota; Kobe U.; Japan
Majima; Keio U.; Japan
Morrison; NIST; USA

$\begin{array}{cr}1, v & 293-433 \\ 1 & 156-470 \\ 1, v & 330-440 \\ 1 & 258-323 \\ \text { v } & 303-333 \\ \text { v } & 323-423 \\ \text { v } & 290-420 \\ 1 & 268-378\end{array}$

$\begin{array}{ll}0.1-16 & 500 \\ \text { sat }-30 & 300\end{array}$

$0.1 \%$

$0.1 \%$

2-12 300

$0.5-1.5 \%$

$0.5-2$--

$0.15 \%$

0.1-sat $\quad 30$

$3 \%$

$0.1-10 \quad 76$

$0.4 \%$

0.1-6 112

$0.1 \%$

$0.1 \%$
.9995
--
$7 / 89-6 / 91$
.999

$\begin{array}{ll}7 / 89-6 / 91 & \\ 10 / 86-9 / 90 & \\ 10 / 90-8 / 91 & \\ \text { comp } & \text { [Yn90] } \\ 9 / 89-8 / 91 & \\ \text { comp } & \text { [Bi90] } \\ \text { comp } & \end{array}$

Virial coefficients

Schramm; Phys. Chem. Inst.;FRG

v 233-296

4

$1 \%$

.9995

.9999

$7 / 89-6 / 91$

$10 / 86-9 / 90$

$.9998 \quad 10 / 90-8 / 91$

sat-7 60

.99

.9998

.999

.999

.999

planned

comp

comp

$--$

comp

Critical parameters

Yin; Xi'an Jiaotong U.; PRC

Bier; U. Karlsrühe; FRG

Schmidt; NIST; USA

$\begin{array}{cc}\mathrm{T}, \mathrm{\rho} & - \\ - & - \\ \mathrm{T}, \mathrm{\rho} & -\end{array}$

- $\quad 15 \mathrm{mK}, 0.5 \%$

.9997

comp

comp

comp

[Yn90]

[Bi90]

-- $\quad 0.01 \mathrm{~g} / \mathrm{cm}^{3} \quad .9997$

[Ch90]

Triple point temperature

Blanke; PTB; FRG

Heat capacity/calorimetry

Nakagawa; Keio U.; Japan

Sami;U. Moncton; Canada

$\begin{array}{ccccc}\mathrm{l} & -- & -- & -- & 0.4 \% \\ \mathrm{l}, \mathrm{v} & 283-353 & 0.5-1.4 & - & 2 \%\end{array}$

.9999

planned

$\begin{array}{ll}-- & 7 / 90--> \\ -- & \text { in prog }\end{array}$

Speed of sound

Kohler; Ruhr U.; FRG

v 230-350
$0.02-0.5 \quad 400 \quad 0.01 \%$

$<15 \quad--\quad 0.5 \%$

-. $\quad ? / 88-? / 91$

Leipertz; U. Erlangen-Nürnberg; FRG 1,v 240-450

$-$

$4 / 90-->$

Dielectric constant

Lehmann; U. Rostock; FRG

Morrison; NIST; USA

$\begin{array}{ccccc}\text { v } & 373-423 & 0.2-3 & 20 & 2 \% \\ \text { v } & 298-433 & 0.04-0.6 & - & 3 \times 10^{-6}\end{array}$


R152a; $\mathrm{CH}_{3} \mathrm{CHF}_{2} ;$ 1,1-difluoroethane (continued)

Property

Investigator

Refractive index

Straub; T.U. München; FRG

Schmidt; NIST; USA

Surface Tension

Okada; Nagaoka U. Tech.; Japan

Schmidt; NIST; USA
Straub; T.U. München; FRG phase range of data no. est. $\mathrm{T}(\mathrm{K}) \quad \mathrm{p}(\mathrm{MPa})$ points accuracy sample purity start/end note dates /ref.

$1, \mathrm{v} \quad 330-440$

2-12 300

$0.05 \%$

.9998

.9997

$10 / 90-8 / 91$

$1, \mathrm{v} \quad 296-384$

sat 40

0.001

comp

[Ch90]

$\begin{array}{lllccccc}1-\mathrm{v} & 230-386 & - & 100 & 0.5 \% & .9998 & 10 / 90-8 / 91 & \\ 1-\mathrm{v} & 273-373 & - & 126 & 0.2 \mathrm{mN} / \mathrm{m} & .9999 & \text { comp } & \text { [Ok90] } \\ \text { l,v } & 296-384 & - & 29 & 0.15 \mathrm{mN} / \mathrm{m} & .9997 & \text { comp } & \text { [Ch90] }\end{array}$

Thermal conductivity

Gross/Hahne; U. Stuttgart; FRG

Hemminger; PTB; FRG

Yata; Kyoto Inst. Tech.; Japan

$\begin{array}{ccccc}1, \mathrm{v} & 260-360 & -- & -- & 1 \% \\ \mathrm{v} & 303-463 & 0.1-0.1 & 7 & 2.5 \% \\ 1 & 250-350 & \text { sat }-30 & -- & --\end{array}$

$1 \quad 293-353$

$1, v \quad 240-450$

$\mathrm{l}, \mathrm{v} \quad 330-440$
$2-8 \quad--\quad i \%$

$<15$

2-12

-.

$1-2 \%$

$0.5-1.5 \%$

rmal diffusivity

Fiebig; Ruhr U.; FRG

Leipertz; U. Erlangen-Nürnberg; FRG

Straub; T.U. München; FRG

Viscosity

Mayinger; T. U. München; FRG

Ström; Chalmers U. Tech.; Swe

Kumagai; Tohoku U.; Japan

Kruse; U. Hannover; FRG

Equation of State

Maurer; U. Kaiserlautern; FRG

Oellrich; U. Karlsrühe; FRG

Huber; NIST; USA

Fischer; ORNL; USA

Gallagher; NIST; USA
$-$
$.997+$
$--$

$? / 90-? / 92$

comp

planned

[Hin89]

comp

$4 / 90-->$

comp

.9998

$-$

2/91-5/91

.99

.999

.999

planned

comp

comp

$\begin{array}{ccccc}\mathrm{v} & 300-425 & 0.1-7.5 & -- & 0.5 \% \\ 1 & 258-323 & 0.5-2 & -- & 2 \% \\ \text { sl } & 273-353 & \text { sat } & 8 & 0.5 \% \\ 1 & 236-353 & ?-2.5 & 8 & 4 \%\end{array}$

$1, v \quad 164-470$

0-50

$1, \mathrm{v}$

$1, \mathrm{v}$ all

$1, \mathrm{v}$

$1, \mathrm{v} \quad 0.5-1.6 \mathrm{~T}_{\mathrm{c}}$
[Km90]

comp

$? / 89-? / 92$

in prog [E190]

comp [Fs90]

comp

[G190]

Ideal Gas Properties

Lucas; U. Duisburg; FRG

- 100-500

0-0

$1 \%$

0.1 in prog

comp
[TR90] 


\section{R152a; $\mathrm{CH}_{3} \mathrm{CHF}_{2} ; 1$,1-difluoroethane (continued)}

Notes and References

[Bi90] K. Bier, R. Hamberger, M. Weisenburger, and J. Zhai; DKV-Jahrestagung 22/23 (1990).

[Ch90] H. B. Chae, J. W. Schmidt, and M. R. Moldover, J. Phys. Chem. (in press).

[E190] J. F. Ely and M. L. Huber, Proc. ASHRAE-Purdue CFC Conference, W. Lafayette, IN, USA, July, 1990, pp $383-392$.

[Fs90] S. K. Fischer and J. Sand, Proc. ASHRAE-Purdue CFC Conference, W. Lafayette, IN, USA, July, 1990, pp $373-382$.

[G190] J. Gallagher, M. McLinden, and G. Morrison, National Institute of Standards and Technology, Standard Reference Data Base 23 (1990).

[Hm89] U. Hammerschmidt and W. Hemminger, 16th DKV-Tagungsbericht 397-410 (1989).

[Km90] A. Kumagai, to be published in Int. J. Thermophysics (1990).

[My90] C. W. Meyer and G. Morrison, J. Phys. Chem. (in press).

[Ok90] M. Okada; report in preparation (1990).

[P189] B. Platzer and G. Maurer, ESCAG: Ein Programmpaket zur Berechnung thermodynamischer Zustandsgrößen reiner Fluide mit Hilfe der Zustandsgleichung von Bender, 16th DKV-Tagungsbericht, Bd. 2, $441-456$ (1989).

[TR90] TRC Thermodynamic Tables, Thermodynamics Research Center, Texas A\&M University, continuously updated; also to be published in J. Phys. Chem. Ref. Data.

[Yn90] J. M. Yin, report in preparation (1990). 
R134; $\mathrm{CHF}_{2} \mathrm{CHF}_{2} ; 1,1,2,2$-tetrafluoroethane

Property

Investigator $\mathrm{T}(\mathrm{K}) \quad \mathrm{p}(\mathrm{MPa})$ points accuracy sample start/end note purity dates /ref.

Vapor pressure

Maezawa; Keio U.; Japan

Tamatsu; Keio U.; Japan

Morrison; NIST; USA 1-v 200-390

$1-\mathrm{v} \quad 303-390$

l-v 268-383

38

34

10
$10 \mathrm{kPa}$

$3 \mathrm{kPa}$

-
.994-.998 comp $.9998 \quad 3 / 90-8 / 90$

.994 comp

[Mz90]

Saturated liquid density

Fukushima; Asahi Glass Co.; Japan

$\begin{array}{ll}\text { sl } & 245-293 \\ \text { sl } & 200-390 \\ \text { sl } & 364-391 \\ \text { sl } & 268-383\end{array}$

$-\quad 7$
$-\quad 38$

7

$0.3 \%$

38

$0.2 \%$

.9994

$.994-.998$

comp

Tatoh; Keio U.; Japan

Morrison; NIST; USA

10

$0.1 \%$

$.998-.999$

comp

10

.994

4/90-->

[Mz90]

Saturated vapor density

Tatoh; Keio U.; Japan

sv $\quad 388-391$

$9 \quad 0.5 \%$

$.998-.999 \quad 4 / 90-->$

Pressure-volume-temperature

Vacek; Czech T.U.; Czech.

Maezawa; Keio U.; Japan

Tamatsu; Keio U.; Japan

$\begin{array}{cr}l & 200-300 \\ 1 & 280-320 \\ l, v & 345-443\end{array}$

$\begin{array}{ccc}\text { sat-55 } & -- & 0.1 \% \\ \text { sat-2 } & 5 & 0.2 \% \\ 0.1-10 & 67 & 0.1 \%\end{array}$
-- $\quad 6 / 91-12 / 9$
$.994-.998$ comp
$.9998 \quad 3 / 90-8 / 90$
.994
comp

[Mz90]

Critical parameters

Tatoh; Keio U.; Japan

Schmidt; NIST; USA

\section{$\mathrm{T}, \rho$}

- $\quad 10 \mathrm{mK}, 0.5 \%$.998-.999 4/90-->

$\mathrm{T}, \rho$

-- $0.01 \mathrm{~g} / \mathrm{cm}^{3}$

[Ch90]

Dielectric constant

Morrison; NIST; USA

v 298-433

0.04-0.5

$3 \times 10^{-6}$

.994

comp

[My90]

Refractive index

Chae; Korea Std. Res. Lab.; Korea

$1, \mathrm{v} \quad 230-456$

$0.1-5$

$-$

$1.0 \%$

.99

planned

$1, \mathrm{v} \quad 293-391$

sat

22

0.001

.994

comp

[Ch90]

Surface Tension

Chae; Korea Std. Res. Lab.; Korea

1-v 230-390

$\begin{array}{ll}-- & 1.0 \%\end{array}$

.99

planned

$1, v \quad 293-391$

$17 \quad 0.15 \mathrm{mN} / \mathrm{m}$

.994

comp

[Ch90]

Thermal conductivity

Chae; Korea Std. Res. Lab.; Korea

$1, v \quad 230-500$

$0.1-5$

$1.0 \%$

.99

3/90-->

Equation of State

Morgenstern; HFV Dresden; FRG

$1, \mathrm{v} \quad 220-480$

$0-4$

$\mathrm{l}, \mathrm{v}$ all

all

Fischer; ORNL. USA

Gallagher; NIST; USA

$1, \mathrm{v}$

$1, \mathrm{v} \quad 0.5-1.6 \mathrm{~T}_{\mathrm{c}}$

$0-2 \mathrm{P}_{\mathrm{c}}$

comp

in prog comp

[E190]

comp

[Fs90]

[Gl90]

Ideal Gas Properties

Lucas; U. Duisburg; FRG

- $100-500$

$\begin{array}{ccc}0-0 & - & 1 \% \\ 0.1 & - & --\end{array}$

in prog

- $0-1500$

comp

[TR90] 
Notes and References

[Ch90] H. B. Chae, J. W. Schmidt, and M. R. Moldover, J. Phys. Chem. (in press).

[E190] J. F. Ely and M. L. Huber, Proc. ASHRAE-Purdue CFC Conference, W. Lafayette, IN, USA, July, 1990, pp 383-392.

[Fs90] S. K. Fischer and J. Sand, Proc. ASHRAE-Purdue CFC Conference, W. Lafayette, IN, USA, July, 1990, pp 373-382.

[G190] J. Gallagher, M. McLinden, and G. Morrison, National Institute of Standards and Technology, Standard Reference Data Base 23 (1990).

[My90] C. W. Meyer and G. Morrison, J. Phys. Chem. (in press).

[Mz90] Y. Maezawa, submitted to J. Chem. Eng. Data (1990).

[TR90] TRC Thermodynamic Tables, Thermodynamics Research Center, Texas A\&M University, continuously updated; also to be published in J. Phys. Chem. Ref. Data. 
Property

Investigator phas $\mathrm{T}(\mathrm{K})$ range of data
$\mathrm{K}) \quad \mathrm{p}(\mathrm{MPa})$ no. est.

points accuracy sample

start/end purity dates /ref.

note

Vapor pressure

Fukushima; Asahi Glass Co.; Japan Morrison; NIST; USA

$\begin{array}{ccccccc}\mathrm{l}-\mathrm{v} & - & - & -- & 5 \mathrm{kPa} & .9997 & 7 / 90-3 / 91 \\ \mathrm{l}-\mathrm{v} & 273-373 & - & 25 & - & .998 & \text { in prog }\end{array}$

Saturated liquid density

$\begin{array}{llllllll}\text { Fukushima; Asahi Glass Co.; Japan } & \text { sl } & 250-310 & - & 8 & 0.3 \% & .9997 & \text { comp }\end{array}$

Pressure-volume-temperature

Fukushima; Asahi Glass Co.; Japan

$1, \mathrm{v} \quad 358-?$

$0.1-6$

$-\quad 0.2 \%$

.9997

$7 / 90-3 / 91$

Sandarusi; NIST; USA

$1, \mathrm{v} \quad 318-473$

$1.4-40 \quad 400 \quad 0.1-0.3 \%$

$.999+$ planned

Critical parameters

Fukushima; Asahi Glass Co.; Japan

$\mathrm{T}, \mathrm{\rho}$

$-$

- $\quad 5 \mathrm{mK}, 0.5 \%$

$.9997 \quad 6 / 90-12 / 90$

Dielectric constant

Morrison; NIST; USA

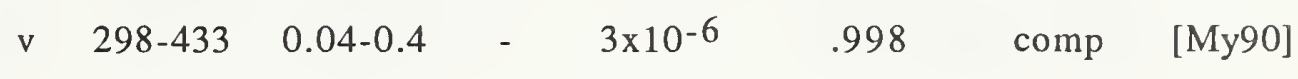

Thermal conductivity

Yata; Kyoto Inst. Tech.; Japan

Perkins; NIST; USA

$1 \quad 250-350$

sat -30

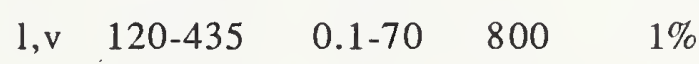

-- planned

-- 1/91-9/92

Thermal diffusivity

Perkins; NIST; USA

$\mathrm{l}, \mathrm{v} \quad 120-435$

$0.1-70 \quad 800$

$5 \%$

$-\quad 1 / 91-9 / 92$

Viscosity

Snelson; NRC; Canada

sl 253-353

$15 \quad 2.5-3.5 \%$

$3 / 91-10 / 91$

Equation of State

Morgenstern; HFV Dresden; FRG

$1, v \quad 240-390$

$0-3.5$

$\mathrm{l}, \mathrm{v}$ all

all

Fischer; ORNL; USA

Gallagher; NIST; USA

$1, v$

$1, \mathrm{v} \quad 0.5-1.6 \mathrm{~T}_{\mathrm{c}} \quad 0-2 \mathrm{P}_{\mathrm{c}}$ comp
in prog
comp
comp

in prog
comp

[E190]

[Fs90]

[G190]

[TR90]

Ideal Gas Properties

Lucas; U. Duisburg; FRG

- $100-500$

$0-0 \quad-\quad 1 \%$

Notes and References

[E190] J. F. Ely and M. L. Huber, Proc. ASHRAE-Purdue CFC Conference, W. Lafayette, IN, USA, July, 1990, pp 383-392.

[Fs90] S. K. Fischer and J. Sand, Proc. ASHRAE-Purdue CFC Conference, W. Lafayette, IN, USA, July, 1990, pp 373-382.

[G190] J. Gallagher, M. McLinden, and G. Morrison, National Institute of Standards and Technology, Standard Reference Data Base 23 (1990).

[My90] C. W. Meyer and G. Morrison, J. Phys. Chem. (in press).

[TR90] TRC Thermodynamic Tables, Thermodynamics Research Center, Texas A\&M University, continuously updated; also to be published in J. Phys. Chem. Ref. Data. 
Property

Investigator phase range of data no. est. $\mathrm{T}(\mathrm{K}) \quad \mathrm{p}(\mathrm{MPa})$ points accuracy sample purity start/end dates note /ref.

Vapor pressure

Blanke; PTB; FRG

LeNeindre; U. Paris Nord; France

Straub; T.U. München; FRG

Fukushima; Asahi Glass Co.; Japan

Maezawa; Keio U.; Japan

Kumagai; Keio U.; Japan

Bier; U. Karlsrühe; FRG

Morrison; NIST; USA

$\begin{array}{lc}l-v & 142-410 \\ l-v & 298-? \\ l-v & -- \\ l-v & 335-410 \\ 1-v & 320-400 \\ l-v & 297-410 \\ l-v & 203-410 \\ l-v & 268-383\end{array}$

Saturated liquid density

Fukushima; Asahi Glass Co.; Japan

Maezawa; Keio U.; Japan

Tanikawa; Keio U.; Japan

Kumagai; Keio U.; Japan

Morrison; NIST; USA

Saturated vapor density

Tanikawa; Keio U.; Japan

Kumagai; Keio U.; Japan

Pressure-volume-temperature

Blanke; PTB; FRG

LeNeindre; U. Paris Nord; France

Nieto de Castro; U. Lisbon; Por

Straub; T.U. München; FRG

Ström; Chalmers U. Tech.; Swe

Fukushima; Asahi Glass Co.; Japan

Maezawa; Keio U.; Japan

Kumagai; Keio U.; Japan

Virial coefficients

Schramm; Phys.-Chem. Inst.; FRG

$\begin{array}{ll}\text { s } 1 & 252-410 \\ \text { s } 1 & 210-400 \\ \text { s } 1 & 353-410 \\ \text { sl } & 346-410 \\ \text { sl } & 268-383\end{array}$

36

$0.2 \%$

$0.2 \%$

9

$0.1 \%$

7

$0.1 \%$

10

sv $\quad 395-410$

sv $357-410$

9

$0.5 \%$

$0.1 \%$

.999
$.998-.9998$

comp

comp

[Km90]

$\begin{array}{ccc}\text { sat-30 } & 300 & 0.1 \% \\ 0.1-40 & -- & -- \\ 0-20 & 50 & 1 \% \\ 2-12 & 300 & -- \\ 0.5-2 & -- & 0.15 \% \\ 1-6 & -- & 0.2 \% \\ \text { sat-2 } & 6 & 0.2 \% \\ 0.3-9 & 70 & 0.1 \%\end{array}$

.9999

--

.995

.999

.99

.9998

.998

$.998-.9998$
[Mz90]

[Km90]

[Bi90]

comp

comp
4/90-10/90

comp

comp

comp

comp

\author{
[Km90] \\ Mz90]
}

$x^{2}$ 
Property

Investigator phase range of data no. est. $\mathrm{T}(\mathrm{K}) \quad \mathrm{p}(\mathrm{MPa})$ points accuracy sample purity start/end dates

note

Speed of sound

Kohler; Ruhr U.; FRG

v 230-350

$\begin{array}{cc}0.025-0.5 & 400 \\ <15 & -- \\ 0.1-100 & -- \\ 0-4 & 50\end{array}$

$0.01 \%$

$0.5 \%$

LeNeindre; U. Paris Nord; France

Trusler; Imperial Col.; UK

Dielectric constant

Nieto de Castro; U. Lisbon; Por

$1, \mathrm{v} \quad-\cdots$

$0-20$

$--$

$0.5 \%$

.995

$12 / 89-10 / 93$

Refractive index

Straub; T.U. München; FRG

Schmidt; NIST; USA

$\begin{array}{ll}l, v & 360-460 \\ l, v & 296-409\end{array}$

-

300

$0.05 \%$

0.001

.9998

.9997

$10 / 90-8 / 91$

comp

[Ch90]

Surface Tension

Straub; T.U. München; FRG

Schmidt; NIST; USA

l-v 230-410

l,v 296-409

$-$

100

$0.5 \%$

.9998

10/90-8/91

$390.15 \mathrm{mN} / \mathrm{m}$

.9997

comp

[Ch90]

Thermal conductivity

LeNeindre; U. Paris Nord; France

$\begin{array}{cc}\mathrm{l} & 298-523 \\ \mathrm{l}, \mathrm{v} & 290-504 \\ \mathrm{v} & 273-423 \\ \mathrm{l} & 250-350 \\ \mathrm{v} & 293-353 \\ \mathrm{l}, \mathrm{v} & 160-450 \\ \mathrm{l}, \mathrm{v} & 203-393\end{array}$

$0.1-70$

$--$

$1-2 \%$

$0-20$

50

$2 \%$

Stephan/Taxis; U. Stuggart; FRG

$<5$

100

sat -30

100

Yata; Kyoto Inst. Tech.; Japan

Tanaka; Kobe U.; Japan

Perkins; NIST; USA

$0.1-$ sat

$0.1-70$

21

$0.1-70$

800

$1 \%$

$--$

$2 \%$

$1 \%$

Venart; U. New Brunswick; Canada

$1, \mathrm{v} \quad 203-393$

$1 \quad 293-353$

2-8

$<15$

$--$

$1--$

$2-12$

300

$\mathrm{l}, \mathrm{v} \quad 360-460$

$0.1-70$

800

$1-2 \%$

$1, v \quad 160-450$

$0.1-70$

Venart; U. New Brunswick; Canada

$0.1-70$

$5 \%$

$5 \%$

Viscosity

Mayinger; T. U. München; FRG

$\begin{array}{ll}\text { v } & 300-384 \\ 1 & 258-323 \\ 1 & 236-353\end{array}$

$0.1-2$

28

$0.5 \%$

$0.5-2$

$2 \%$

Kruse; U. Hannover; FRG

$?-2.5$

$4 \%$

.9991
.99
999

comp
planned
comp

[My90]

.999

comp

Equation of State

Maurer; U. Kaiserlautern; FRG

$\begin{array}{ccc}\mathrm{v} & 257-427 & 0-4 \\ 1, \mathrm{v} & - & - \\ 1, \mathrm{v} & \text { all } & \text { all } \\ \mathrm{l,v} & 0.5-1.6 \mathrm{~T}_{\mathrm{c}} & 0-2 \mathrm{P}_{\mathrm{c}}\end{array}$

$0-4$

comp

$\begin{array}{cc}- & ? / 89-? / 92 \\ - & \text { in prog }\end{array}$

Oellrich; U. Karlsrühe; FRG

Huber; NIST; USA

Gallagher; NIST; USA

$\begin{array}{ll}- & \text { ?/89-?/92 } \\ \text { - } & \text { in prog }\end{array}$

comp

[P190]

[E190]

[G190]

Ideal Gas Properties

Lucas; U. Duisburg; FRG

- $100-500$

$0-0$

$1 \%$

in prog

comp

[TR90] 


\section{R142b; $\mathrm{CClF}_{2} \mathrm{CH}_{3}$; 1-chloro-1,1-difluoroethane (continued)}

\section{References}

[Bi90] K. Bier, R. Hamberger, M. Weisenburger, and J. Zhai; DKV-Jahrestagung 22/23 (1990).

[Ch90] H. B. Chae, J. W. Schmidt, and M. R. Moldover, J. Phys. Chem. (in press).

[Cs90] C. A. Nieto de Castro, paper in preparation.

[E190] J. F. Ely and M. L. Huber, Proc. ASHRAE-Purdue CFC Conference, W. Lafayette, IN, USA, July, 1990, pp 383-392.

[G190] J. Gallagher, M. McLinden, and G. Morrison, National Institute of Standards and Technology, Standard Reference Data Base 23 (1990).

[Km90] K. Kumagai, submitted to J. Chem. Eng. Data (1990).

[My90] F. Mayinger, data to be published September, 1990.

[Mz90] Y. Maezawa, submitted to J. Chem. Eng. Data (1990).

[P190] A. W. Polt, dissertation, Universität Kaiserlautern (19??).

[Tn90] Y. Tanaka, submitted to Int. J. Thermophysics (1990).

[TR90] TRC Thermodynamic Tables, Thermodynamics Research Center, Texas A\&M University, continuously updated; also to be published in J. Phys. Chem. Ref. Data. 
R123; $\mathrm{CHCl}_{2} \mathrm{CF}_{3} ;$ 1,1-dichloro-2,2,2-trifluoroethane

Property

Investigator phase range of data no. est. $\mathrm{T}(\mathrm{K}) \quad \mathrm{p}(\mathrm{MPa})$ points accuracy sample start/end

note purity dates /ref.

Vapor pressure

Baroncini; U. Ancona; Italy

Gorenflo; U. Paderborn; FRG

LeNeindre; U. Paris Nord; France

Straub; T.U. München; FRG

Oguchi; Kanagawa Inst. Tech.; Japan

Kubota; Kobe U.; Japan

Fukushima; Asahi Glass Co.; Japan

Maezarva; Keio U.; Japan

Piao; Keio U.; Japan

Qian; Keio U.; Japan

Mingshan; Tsinghua U.; PRC

Magee; NIST; USA

Weber; NIST; USA

Morrison; NIST; USA

Morrison; NIST; USA

$\begin{array}{lc}\text { l-v } & 240-360 \\ \text { l-v } & 293-423 \\ \text { l-v } & 298-? \\ \text { l-v } & 360-460 \\ \text { l-v } & 243-456 \\ \text { l-v } & 273-457 \\ \text { l-v } & 314-456 \\ \text { l-v } & 280-350 \\ \text { l-v } & 310-456 \\ \text { l-v } & 310-420 \\ \text { l-v } & 248-358 \\ \text { l-v } & 166-400 \\ \text { l-v } & 273457 \\ \text { l-v } & 268-383 \\ \text { l-v } & 273-373\end{array}$

s1 295-423

sl 248-423

sl 254-433

sl 281-456

sl 200-400

s $1 \quad 300-420$

sl 401-456

sl $300-457$

sl 268-383

Morrison; NIST; USA

Saturated vapor density

Gorenflo; U. Paderborn; FRG

Fukushima; Asahi Glass Co.; Japan

Tanikawa; Keio U.; Japan

Weber; NIST; USA s v 293-423

sv $352-456$

sv $438-456$

sv $433-457$

$\begin{array}{ccccc}-- & 0.5 \% & -- & 7 / 90-6-91 & \\ 27 & 0.04 \% & .95-.97 & -- & \\ -- & -- & -- & 10 / 90--> & \\ 50 & 0.5-1.5 \% & .998 & 7 / 90-12 / 90 & \\ 45 & 0.5 \mathrm{kPa} & .998 & \text { comp } & \\ 38 & 3 \mathrm{kPa} & .995 & \text { comp } & \text { [Kb89] } \\ 65 & 5 \mathrm{kPa} & .998-.999 & \text { comp } & \\ 8 & 7 \mathrm{kPa} & .9993 & \text { comp } & \text { [Mz90] } \\ 67 & 2 \mathrm{kPa} & .998-.9993 & ? / 89-? / 91 & \\ 36 & 1 \mathrm{kPa} & .996-.99945 / 90-7 / 90 & \\ 20 & 0.02 \% & -- & \text { planned } & \\ -- & 0.01-0.05 \% & .9999 & 5 / 91-9 / 91 & \\ 64 & 0.05 \% & .9995 & \text { comp } & \text { [Wb90] } \\ 15 & - & .9999 & \text { comp } & \text { [Mo90] } \\ 25 & - & .9999 & \text { in prog } & \end{array}$

$\begin{array}{ccccc}27 & 0.04 \% & .95-.97 & -- & \\ 24 & 0.2-0.3 \% & .996-.999 & \text { comp } & \text { [Yk88] } \\ 17 & 0.1 \% & .998 & \text { comp } & \\ 35 & 0.3 \% & .998-.999 & \text { comp } & \\ 23 & 0.2 \% & .9993 & \text { comp } & \text { [Mz90] } \\ 5 & 0.15 \% & .998-.9993 & ? / 89-? / 91 & \\ 10 & 0.1-0.5 \% & .998-.9999 & \text { comp } & \\ - & 0.05 \% & .9995 & \text { comp } & \text { [Wb90] } \\ 10 & - & .9999 & \text { comp } & {[\mathrm{Mo} 90]}\end{array}$

$\begin{array}{cccc}27 & 0.2 \% & .95-.97 & -- \\ 27 & 0.3 \% & .998-.999 & \text { comp } \\ 9 & 0.1-0.5 \% & .998-.9999 & \text { comp } \\ 5 & 0.05 \% & .9995 & \text { comp }\end{array}$

[Wb90] 
Property

Investigator phase rang of data $\mathrm{p}(\mathrm{MPa})$ points accuracy sample purity start/end dates note

/ref.

Pressure-volume-temperature

Baroncini; U. Ancona; Italy

LeNeindre; U. Paris Nord; France

Nieto de Castro; U. Lisbon; Por.

Straub; T.U. München; FRG

Oguchi; Kanagawa Inst. Tech.; Japan

Matsuo; Kobe U.; Japan

Matsuo; Kobe U.; Japan

Fukushima; Asahi Glass Co.; Japan

Maezawa; Keio U.; Japan

Piao; Keio U.; Japan

Qian; Keio U.; Japan

Woolf; Australian Nat. U.; Aus.

Mingshan; Tsinghua U.; PRC

Morrison; NIST; USA

Weber; NIST; USA

$\begin{array}{cc}\mathrm{v} & 240-360 \\ \mathrm{l}, \mathrm{v} & 298-413 \\ \mathrm{l}, \mathrm{v} & <\mathrm{T}_{\mathrm{c}} \\ \mathrm{l}, \mathrm{v} & 360-460 \\ \mathrm{l} & 243-493 \\ \mathrm{l} & 293-323 \\ \mathrm{l} & 293-353 \\ \mathrm{l}, \mathrm{v} & 352-484 \\ \mathrm{l} & 280-340 \\ \mathrm{l}, \mathrm{v} & 310-525 \\ \mathrm{v} & 310-420 \\ \mathrm{l} & 273-338 \\ \mathrm{v} & 248-358 \\ \mathrm{l} & 268-378 \\ \mathrm{v} & 338-453\end{array}$

$\begin{array}{ccc}0.1-\text { sat } & -- & 0.5 \% \\ 0.1-100 & -- & -- \\ <20 & -- & 0.3 \% \\ 0.7-12 & 300 & 0.5-1.5 \% \\ 0.07-16 & 27 & 0.01 \% \\ \text { sat-40 } & 68 & 0.04 \% \\ \text { sat-40 } & 44 & 0.02 \% \\ 1-5 & 59 & 0.2 \% \\ \text { sat-2 } & 16 & 0.2 \% \\ 0.5-12 & 134 & 0.15 \% \\ 0.1-\text { sat } & 65 & 0.2-0.7 \% \\ \text { sat-400 } & -- & 0.1-0.5 \% \\ 0.1-6 & 80 & 0.2 \% \\ \text { sat-7 } & 60 & 0.1 \% \\ 0-\text { sat } & 75 & 0.05 \%\end{array}$

$-$

7/90-6-91

-- 10/90-->

$.995 \quad 12 / 89-10 / 93$

$.998 \quad 7 / 90-12 / 90$

comp

.999

comp

.999

comp

$.998-.999$

comp

.9993

comp

$.998-.9993 ? / 89-? / 91$

$.996-.99945 / 90-7 / 90$

[Ko90]

[Ko88]

[Mz90]

Virial coefficients

Schramm; Phys.-Chem. Inst.; FRG

v $268-296$

2

$5 \%$

-- comp

Critical parameters

LeNeindre; U. Paris Nord; France

Straub; T.U. München; FRG

Kubota; Kobe U.; Japan

$\begin{array}{cc}-- & - \\ -- & - \\ \mathrm{T}, \mathrm{P} & \\ \mathrm{T}, \rho & - \\ \mathrm{P} & - \\ \mathrm{T}, \rho & - \\ \mathrm{T}, \mathrm{P}, \mathrm{\rho} & -\end{array}$

Heat capacity/calorimetry

Nakagawa; Keio U.; Japan

Magee; NIST; USA

$1 \quad 276-440$

sat-3 $\quad 80$

$0.4 \%$

$0.5 \%$

.9982

8/90-->

$-$

.9999

.9995

planned

comp

comp

[Wb90]

$1 \quad 166-340$

sat -35

-- $\quad 0.5-1.5 \%$

998

$10 / 90-->$

$50 \mathrm{mK}, 3 \mathrm{kPa}$

.995

$7 / 90-12 / 90$

$25 \mathrm{mK}$

$.998-.999$

comp

[Kb89]

$2 \mathrm{kPa}$

$.998-.9993$

comp

$10 \mathrm{mK}, 0.5 \% .998-.9999$

/89-?91

$0.05 \%$

.9995

comp

[Wb90]

Speed of sound

Leipertz; U. Erlangen-Nürnberg; FRG

I,v $\quad 240-450$

LeNeindre; U. Paris Nord; France

$1, \mathrm{v} \quad 298-523$

Trusler; Imperial Col.; UK

Takagi; Kyoto Inst. Tech.; Japan

Mingshan; Tsinghua U.; PRC

Goodwin; NIST; USA

$\begin{array}{lc}v & -- \\ l & 280-370\end{array}$

$<15$

$-$

$0.5 \%$

$0.1-100 \quad--\quad--$

$0-4 \quad 50$

$0.005 \%$

sat-75 $\quad 170$

$0.2 \%$

v 248-358

0.1-1.5 --

$0.2 \%$

0.02-0.07 49

$0.01 \%$

.9999

comp

$5 / 91-9 / 91$

Dielectric constant

Nieto de Castro; U. Lisbon; Por.

$\begin{array}{cc}\mathrm{l}, \mathrm{v} & <\mathrm{Tc} \\ \mathrm{I} & 298-313 \\ \mathrm{v} & 298-433\end{array}$

$\begin{array}{ccc}<20 & -- & 0.5 \% \\ \text { sat-35 } & 32 & 1 \% \\ 0.04-0.1 & - & 3 \times 10^{-6}\end{array}$

.995

Tanaka; Kobe U.; Japan

Morrison; NIST; USA

.999

.9999

12/89-10/93

$4 / 90-->$
$10 / 90-->$
$10 / 90-->$
comp
planned
in prog

comp

[Ko90]

[Me90] 
R123; $\mathrm{CHCl}_{2} \mathrm{CF}_{3} ;$ 1,1-dichloro-2,2,2-trifluoroethane (continued)

Property

Investigator

Refractive index

Straub; T.U. München; FRG

Chae; Korea Std. Res. Lab.; Korea

$1, \mathrm{v} \quad 360-460$

$\mathrm{l}, \mathrm{v} \quad 230-456$

$0.7-12 \quad 300$

$0.1-5 \quad--$

$0.05 \%$

$1.0 \%$

1-v 223-457

l-v 273-290

l-v 237-443

$1-v \quad 230-456$

l-v 263-358

l-v 253-423
Straub; T.U. München; FRG

Higashi; Iwaki Meisei U.; Japan

Okada; Nagaoka U. Tech.; Japan

Chae; Korea Std. Res. Lab.; Korea

Mingshan; Tsinghua U.; PRC

Schmidt; NIST; USA

Thermal conductivity

Assael; Aristotle U.; Greece
Gross/Hahne; U. Stuggart; FRG
LeNeindre; U. Paris Nord; France
Nieto de Castro; U. Lisbon; Por.
Yata; Kyoto Inst. Tech.; Japan
Tanaka; Kobe U.; Japan
Matsuo; Kobe U.; Japan
Nagasaka; Keio U.; Japan
Chae; Korea Std. Res. Lab.; Korea
Perkins; NIST; USA
Venart; U. New Brunswick; Canada

$\begin{array}{ll}l, v & 230-370 \\ l, v & 260-364\end{array}$

$1, \mathrm{v} \quad 298-523$

$1, \mathrm{v}<\mathrm{T}_{\mathrm{c}}$

$1 \quad 250-350$

$1 \quad 283-323$

$1 \quad 298-323$

$1 \quad 273-353$

$1, \mathrm{v} \quad 230-500$

$\mathrm{l}, \mathrm{v} \quad 180-450$

l,v 203-393

$\begin{array}{ccc}<40 & -- & 0.5 \% \\ 0.1-8.0 & 65 & 1 \% \\ 0.1-100 & -- & -- \\ <20 & -- & 0.5 \% \\ \text { sat-30 } & 127 & -- \\ \text { sat-200 } & 36 & 1.0 \% \\ \text { sat-100 } & 33 & 1 \% \\ \text { sat-40 } & -- & 0.5 \% \\ 0.1-5 & -- & 1.0 \% \\ 0.1-70 & 800 & 1 \% \\ 0.1-70 & -- & 1 \%\end{array}$

$0.1-70$

$\begin{array}{ccc}100 & 0.5 \% & .998 \\ 21 & 0.4 \mathrm{mN} / \mathrm{m} & .9984 \\ 186 & 0.2 \mathrm{mN} / \mathrm{m} & .9984 \\ -- & 1.0 \% & .99 \\ -- & -- & -- \\ 22 & 0.15 \mathrm{mN} / \mathrm{m} & .995\end{array}$

planned

in prog

comp

planned

planned

comp

[Ok90]

[Ch90]

Thermal diffusivity

Fiebig; Ruhr U.; FRG

$1 \quad 273-363$

$1-8$

$1 \%$

$1-2 \%$

Straub; T.U. München; FRG

Perkins; NIST; USA

Venart; U. New Brunswick; Canada

$1, v \quad 240-450$

$<15$

--

$0.5-1.5 \%$

$0.7-12$

300

$1, \mathrm{v} \quad 180-450$

$0.1-70$

800

$5 \%$

$5 \%$

$\begin{array}{cc}-- & 6 / 89-? \\ .995 & ? / 88-? / 92 \\ -- & 10 / 90--> \\ .995 & 12 / 89-10 / 93 \\ .995-.9995 & \text { comp } \\ .999 & \text { comp } \\ .999 & \text { in prog } \\ .998 & ? / 89--> \\ .99 & 3 / 90--> \\ -- & 1 / 91-9 / 92 \\ -- & \text { in prog } \\ & \\ & \\ -- & 8 / 90-12 / 90 \\ -- & 4 / 90--> \\ .998 & 7 / 90-12 / 90 \\ -- & 1 / 91-9 / 92 \\ -- & \text { in prog }\end{array}$

[Gr90]

[Yt89]

[Tn88]

l,v 203-393

$0.1-70$

$\begin{array}{lr}v & 301-573 \\ v & 300-425 \\ v & 323-423 \\ s 1 & 273-353 \\ 1 & 293-313 \\ 1 & 233-418 \\ 1 & 273-338 \\ \mathrm{sl} & 263-358 \\ 1 & 170-320 \\ \mathrm{sl} & 253-353\end{array}$

0.01-0.1 10

$0.5 \%$

0.1-2.1 28

$0.5 \%$

0.1 -sat 48

$0.3 \%$

$$
\text { sat }
$$

$0.5 \%$

sat- 100

$2 \%$

Tanaka; Kobe U.; Japan

Nagashima; Keio U.; Japan

Woolf; Austrlian Nat. U.; Aus.

Mingshan; Tsinghua U.; PRC

Diller; NIST; USA

Snelson; NRC; Canada

sat-20 --

sat $-400 \quad--$

$--$

$0.1-0.5 \%$

sat-35

$--$

60

$15 \quad 2.5-3.5 \%$

$\begin{array}{ccr}-- & 1 / 90--> & \\ .999 & \text { comp } & \text { [My90] } \\ .996 & \text { comp } & \text { [Tk90] } \\ .996 & \text { comp } & {[\mathrm{Km} 90]} \\ .999 & -- & \\ .9977 & \text { ?/89--> } \\ -- & \text { 8/90--> } \\ -- & \text { planned } \\ -- & \text { comp } \\ - & \text { 3/91-10/91 }\end{array}$


Property

Investigator

Equation of State

Maurer; U. Kaiserlautern; FRG

Huber; NIST; USA

Gallagher; NIST; USA

Ideal Gas Properties

Lucas; U. Duisburg; FRG

Marsh; Texas A\&M; USA phase range of data no. est.

$\mathrm{T}(\mathrm{K}) \quad \mathrm{p}(\mathrm{MPa})$ points accuracy

sample

start/end

note

purity dates /ref.

$\begin{array}{ccc}\mathrm{v} & 288-368 & 0-0.7 \\ \mathrm{l}, \mathrm{v} & \text { all } & \text { all } \\ \mathrm{l,v} & 0.5-1.6 \mathrm{~T}_{\mathrm{c}} & 0-2 \mathrm{P}_{\mathrm{c}}\end{array}$

- $100-500$

$0-0$

$1 \%$

in prog

- $\quad 0-1500$

0.1

$--$

comp

[TR90]

\section{Notes and References}

[Ch90] H. B. Chae, J. W. Schmidt, and M. R. Moldover, J. Chem. Eng Data 356 (1990).

[E190] J. F. Ely and M. L. Huber, Proc. ASHRAE-Purdue CFC Conference, W. Lafayette, IN, USA, July, 1990, pp 383-392.

[G190] J. Gallagher, M. McLinden, and G. Morrison, National Institute of Standards and Technology, Standard Reference Data Base 23 (1990).

[Gr90] U. Gross and E. Hahne, to be published in Proc., IIR Commission B1 Meeting, Tel-Aviv, March, 1990.

[Ko88] Kobe University thesis (1988).

[Ko90] Kobe University thesis (1990).

[Kb89] H. Kubota, Int. J. Thermophysics 10629 (1989).

[Km90] A. Kumagai, to be published in Int. J. Thermophysics.

[Me90] C. W. Meyer and G. Morrison, J. Phys. Chem. (in press).

[Mo90] G. Morrison and D. K. Ward, Fluid Phase Equilibria (in press).

[Mz90] Y. Maezawa, J. Chem. Eng. Data, 35 (in press).

[My90] F. Mayinger, data to be published September, 1990.

[Ok90] M. Okada, to be published in Proc., IIR Commission B1 Meeting, Tel-Aviv, March, 1990.

[Tk90] M. Takahashi, to be presented at the 11th Japan Symp. on Thermophysical Properties, November, 1990, Tokyo.

[Tn88] Y. Tanaka, Int. J. Thermophysics, 9465 (1988).

[TR90] TRC Thermodynamic Tables, Thermodynamics Research Center, Texas A\&M University, continuously updated; also to be published in J. Phys. Chem. Ref. Data.

[Yt89] J. Yata, et al., Thermal conductivity of R123 and R134a in liquid phase, Proc. 2nd Asian Thermophysical Properties Conference, p. 201 (1989).

[Yk88] C. Yokoyama, Proc., 29th High Pressure Conference of Japan, p. 116 (1988). 
Property

Investigator phase range of data no. est. $\mathrm{T}(\mathrm{K}) \quad \mathrm{p}(\mathrm{MPa})$ points accuracy sample purity start/end note dates /ref.

Vapor pressure

Otake; Tohoku U.; Japan

Kubota; Kobe U.; Japan

Morrison; NIST; USA

Saturated liquid density

Yokoyama; Tohoku U.; Japan

Morrison; NIST; USA

Pressure-volume-temperature

Otake; Tohoku U.; Japan

Matsuo; Kobe U.; Japan

Woolf; Australian Nat. U.; Aus.

Morrison; NIST; USA

$\begin{array}{llllclll}\text { l-v } & 297-447 & - & 20 & 0.1 \mathrm{kPa} & .998 & \text { comp } & \text { [Ot89] } \\ \text { l-v } & 303-458 & - & 32 & 3 \mathrm{kPa} & .998 & \text { comp } & \text { [Kb89] } \\ \text { l-v } & 268-383 & - & 10 & - & .991 & \text { comp } & \end{array}$

$\begin{array}{lcccccc}\text { sl } & 247-422 & - & 31 & 0.3 \% & .998-.999 & \text { comp } \\ \text { sl } & 268-383 & - & 10 & - & .991 & \text { comp }\end{array}$

[Yk88]

comp

$\begin{array}{cccccccc}\text { v } & 374-465 & 0.5-5 & 19 & 0.5 \% & .998 & \text { comp } & \text { [Ot89] } \\ 1 & 293-333 & \text { sat-40 } & 44 & 0.04 \% & .999 & \text { comp } & \text { [Ko89a] } \\ 1 & 273-338 & \text { sat-400 } & -- & 0.1-0.5 \% & -- & 8 / 90--> & \\ 1 & 268-378 & \text { sat-7 } & 60 & 0.1 \% & .991 & \text { comp } & \end{array}$

Critical parameters

Schmidt; NIST; USA

$\mathrm{T}$,

$-\quad 0.01 \mathrm{~g} / \mathrm{cm}^{3}$

$.991 \quad$ comp

[Ch90]

Speed of sound

Goodwin; NIST; USA

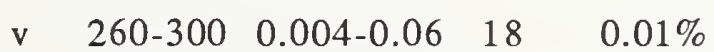

in prog

Dielectric constant

Morrison; NIST; USA

v 298-433

$0.04-0.1 \quad-\quad 3 \times 10^{-6}$

.991

comp

[My90]

Refractive index

Schmidt; NIST; USA

$1, \mathrm{v} \quad 297-460$

sat

40

0.001

.991

comp

[Ch90]

Surface tension

Schmidt; NIST; USA

l,v 297-460

sat

$290.15 \mathrm{mN} / \mathrm{m}$

.991

comp

[Ch90]

Thermal conductivity

Hemminger; PTB; FRG

v $\quad 303-463$

$0.1-0.1$

7

$2.5 \%$

.997

comp

[Hm89]

Viscosity

Takahashi; Tohoku U.; Japan

Kumagai; Tohoku U.; Japan

Tanaka; Kobe U.; Japan

v $323-423$

0.1 -sat

47

$0.3 \%$

$0.5 \%$

sl 273-353

sat

39

$2 \%$

$1 \quad 298-323$

sat- 120

$0.1-0.5 \%$

--
.998
.999

comp

[Tk90]

comp [Km90]

comp [Ko89]

8/90-->

in prog

- $100-500$

$0-0$

$1 \%$

0.1

comp

[TR90] 
Notes and References

[Ch90] H. B. Chae, J. W. Schmidt, and M. R. Moldover, J. Phys. Chem. (in press).

[Hm89] U. Hammerschmidt and W. Hemminger, DKV-Tagungsberict $16397-410$ (1989).

[Kb89] H. Kubota, Int. J. Thermophysics 10629 (1989).

[Km90] A. Kumagai, to be published in Int. J. Thermophysics.

[Ko89] Kobe University thesis (1989).

[Ko89a] Kobe University thesis (1989).

[My90] C. W. Meyer and G. Morrison, J. Phys. Chem. (in press).

[Ot89] K. Otake, Proc., 30th High Pressure Conference of Japan, p356 (1989).

[Tk90] M. Takahashi, to be presented at the 11th Japan Symp. on Thermophysical Properties, November, 1990, Tokyo.

[TR90] TRC Thermodynamic Tables, Thermodynamics Research Center, Texas A\&M University, continuously updated; also to be published in J. Phys. Chem. Ref. Data.

[Yk88] C. Yokoyama, Proc., 29th High Pressure Conference of Japan, p. 116 (1988). 
R141b; $\mathrm{CCl}_{2} \mathrm{FCH}_{3} ; 1$,1-dichloro-1-fluoroethane

Property

Investigator phase range of data no. est. $\mathrm{T}(\mathrm{K}) \quad \mathrm{p}(\mathrm{MPa})$ points accuracy sample purity start/end dates

note

/ref.

Vapor pressure

LeNeindre; U. Paris Nord; France

Kubota; Kobe U.; Japan

Maezawa; Keio U.; Japan

Holste; Texas A\&M U.; USA

Weber; NIST; USA

Morrison; NIST; USA

Saturated liquid density

Kumagai; Tohoku U.; Japan

Fukushima; Asahi Glass Co.; Japan

Maezawa; Keio U.; Japan

Holste; Texas A\&M U.; USA

Morrison; NIST; USA

Morrison; NIST; USA

Saturated vapor density

Holste; Texas A\&M U.; USA

Pressure-volume-temperature

LeNeindre; U. Paris Nord; France

Nieto de Castro; U. Lisbon; Por.

Matsuo; Kobe U.; Japan

Kubota; Kobe U.; Japan

Maezawa; Keio U.; Japan

Woolf; Australian Nat. U.; Aus.

Holste; Texas A\&M U.; USA

Weber; NIST; USA

Morrison; NIST; USA

Critical parameters

LeNeindre; U. Paris Nord; France

Holste; Texas A\&M U.; USA

Schmidt; NIST; USA

Speed of sound

LeNeindre; U. Paris Nord; France

Trusler; Imperial Col.; UK

Goodwin; NIST; USA

Dielectric constant

Nieto de Castro; U. Lisbon; Por.

Morrison; NIST; USA

$\begin{array}{ll}l-v & 298-480 \\ l-v & 278-373 \\ l-v & 200-400 \\ l-v & 250-400 \\ l-v & 273-418 \\ l-v & 268-383\end{array}$

sl 273-353

sl 245-369

sl 200-400

sl $250-400$

sl 268-383

sl 273-373

sv $\quad 250-400$

$\begin{array}{cc}- & 9 \\ - & -- \\ - & 37 \\ - & - \\ - & 10 \\ - & 25\end{array}$

$0.2 \%$

$0.3 \%$

$0.2 \%$

$0.1 \%$

-

$-$

$0.1 \%$

$$
\begin{array}{cc}
\mathrm{l,v} & 298-413 \\
\mathrm{l,v} & <\mathrm{T}_{\mathrm{C}} \\
\mathrm{l} & 293-348 \\
\mathrm{l}, \mathrm{v} & 298-323 \\
\mathrm{l} & 280-400 \\
\mathrm{l} & 273-338 \\
\mathrm{l}, \mathrm{v} & 250-400 \\
\mathrm{v} & 329-418 \\
\mathrm{l} & 268-378
\end{array}
$$

$\begin{array}{cc}0.1-100 & -- \\ <20 & -- \\ \text { sat-100 } & 111 \\ 0.1-200 & 63 \\ \text { sat-2 } & 24 \\ \text { sat-400 } & -- \\ 0-70 & -- \\ 0-s a t & 50 \\ \text { sat-7 } & 60\end{array}$

-.

$0.3 \%$

$0.05 \%$

$0.1-0.3 \%$

$0.2 \%$

$0.1-0.5 \%$

$0.1 \%$

$0.05 \%$

$0.1 \%$
.995

.9947

.988

-

.9994

.9994

$9 / 90-10 / 91$

10/90-->

comp

comp

9/90-10/91

comp

comp

comp $[\mathrm{Km} 90]$
comp
comp [Mz90]
$9 / 90-10 / 91$
comp
in prog

10/90-->

$.995 \quad 12 / 89-10 / 93$

.999

.999

.988

$--$

$--$

.9994

.9994 comp [Ko90]

?/89-->

comp

8/90-->

9/90-10/91

comp

comp

$$
\begin{array}{ccccccc}
- & - & - & -- & -- & -- & 10 / 90--> \\
\mathrm{T}, \mathrm{P}, \mathrm{p} & - & - & -- & 0.1 \% & -- & 9 / 90-10 / 91 \\
\mathrm{~T}, \rho & - & - & -- & 0.01 \mathrm{~g} / \mathrm{cm}^{3} & .9995 & \text { comp }
\end{array}
$$

[Ch90]

$\begin{array}{ccccccc}\mathrm{l}, \mathrm{v} & 298-523 & 0.1-100 & -- & -- & -- & 10 / 90--> \\ \mathrm{v} & -- & 0-4 & 50 & 0.005 \% & -- & 10 / 90--> \\ \text { v } & 260-315 & 0.004-0.05 & 45 & 0.01 \% & .9994 & \text { in prog }\end{array}$

$\begin{array}{cccccccc}1, \mathrm{v} & <\mathrm{Tc} & <20 & -- & 0.5 \% & .995 & 12 / 89-10 / 93 \\ \mathrm{v} & 298-433 & 0.04-0.1 & - & 3 \times 10^{-6} & .9994 & \text { comp } & \text { [My90] }\end{array}$

$\begin{array}{lllll}1, \mathrm{v} & 323-476 & \text { sat } & 40 & 0.00\end{array}$

$.9995 \quad$ comp

[Ch90] 
Property

Investigator

Surface tension

Schmidt; NIST; USA

Thermal conductivity

LeNeindre; U. Paris Nord; France

Nieto de Castro; U. Lisbon; Por.

Wakeham; Imperial Col.; UK

Yata; Kyoto Inst. Tech.; Japan

Tanaka; Kobe U.; Japan

Matsuo; Kobe U.; Japan

Perkins; NIST; USA

Venart; U. New Brunswick; Canada phase

range of data $\mathrm{T}(\mathrm{K})$

$\mathrm{p}(\mathrm{MPa})$ points accuracy

sample

start/end note

purity dates /ref.

$$
1, \mathrm{v} \quad 323-476
$$

$28 \quad 0.15 \mathrm{mN} / \mathrm{m}$

.9995

comp

[Ch90]

\begin{tabular}{cccccccc} 
l,v & $298-523$ & $0.1-100$ & -- & -- & -- & \multicolumn{2}{c}{$10 / 90->$} \\
l,v & $<\mathrm{T}_{\mathrm{c}}$ & $<20$ & -- & $0.5 \%$ & .995 & $12 / 89-10 / 93$ & \\
sl,sv & $260-370$ & sat & 20 & -- & .999 & comp & [Wk90] \\
l & $250-350$ & sat-30 & -- & -- & $.995-.9995$ & -- & \\
v & $293-353$ & $0.1-0.1$ & 4 & $2 \%$ & .999 & comp & [Tn90] \\
l & $298-313$ & sat-60 & 13 & $1 \%$ & .999 & in prog & \\
l,v & $180-450$ & $0.1-70$ & 800 & $1 \%$ & -- & $1 / 91-9 / 92$ & \\
l,v & $203-393$ & $0.1-70$ & -- & $1 \%$ & -- & planned &
\end{tabular}

Thermal diffusivity

Perkins; NIST; USA

Venart; U. New Brunswick; Canada

l,v $\quad 180-450$

0.1-70 800

$5 \%$

$0.1-70 \quad--\quad 5 \%$

$\begin{array}{cc}-- & 1 / 91-9 / 92 \\ -- & \text { planned }\end{array}$

Viscosity

Kumagai; Tohoku U.; Japan

Tanaka; Kobe U.; Japan

l,v 203-393

sl 273-353

$\begin{array}{ccc}\text { sat } & 9 & 0.5 \% \\ \text { sat-120 } & 39 & 2 \% \\ \text { sat-400 } & -- & 0.1-0.5 \% \\ \text { sat-35 } & 60 & 3 \%\end{array}$

.995
.999
--
--

comp

[Km90]

comp

8/90-->

comp

Diller; NIST; USA

Equation of State

Morgenstern; HFV Dresden; FRG

Huber; NIST; USA

$$
\begin{array}{lc}
\mathrm{l,v} & 273-520 \\
\mathrm{l,v} & \text { all }
\end{array}
$$

$0-4$

all

comp

in prog

[E190]

Ideal Gas Properties

Lucas; U. Duisburg; FRG

- $100-500$

$0-0$

$1 \%$

in prog

Marsh; Texas A\&M; USA

- $0-1500$

0.1

comp

[TR90]

Notes and References

[Ch90] H. B. Chae, J. W. Schmidt, and M. R. Moldover, J. Phys. Chem. (in press).

[E190] J. F. Ely and M. L. Huber, Proc. ASHRAE-Purdue CFC Conference, W. Lafayette, IN, USA, July, 1990, pp 383-392.

[Km90] A. Kumagai, to be presented at the 11th Japan Symp. on Thermophysical Properties, November, 1990, Tokyo.

[Ko90] Kobe University thesis (1990).

[My90] C. W. Meyer and G. Morrison, J. Phys. Chem. (in press).

[Mz90] Y. Maezawa, submitted to J. Chem. Eng. Data (1990)

[Tn90] Y. Tanaka, submitted to Int. J. Thermophysics (1990).

[TR90] TRC Thermodynamic Tables, Thermodynamics Research Center, Texas A\&M University, continuously updated; also to be published in J. Phys. Chem. Ref. Data.

[Wk90] W. Wakeham, data presented in proprietary report (1990). 


\section{Other Pure Fluids}

Fluid/Investigator/

/Property phase

range of data

no.

est. $\mathrm{T}(\mathrm{K})$ sample purity start/end dates note /ref.

R227

Gorenflo; U. Paderborn; FRG

vapor pressure

sat. liquid density

sat. vapor density l-v 293-375

sl 293-370

sv $293-370$

\section{7}

17

17
$0.04 \%$

$0.04 \%$

$0.2 \%$
.995

.995

.995 in prog

in prog

in prog

R124a

Morgenstern; HFV Dresden; FRG

equation of state

l,v $\quad 243-450$

$0-3.5$

comp

[Mr90]

R225ca

Widiatmo; Keio U.; Japan

vapor pressure

saturation density

pressure-volume-temperature

Higashi; Iwaki Meisei U.; Japan surface tension

$$
\begin{array}{cc}
\mathrm{l-v} & \mathrm{T}_{\mathrm{tp}}-400 \\
\mathrm{sl} & \mathrm{T}_{\mathrm{tp}}-400 \\
1 & 280-400
\end{array}
$$

l-v 273-320
-- $\quad 10 \mathrm{kPa}$

-. $\quad 0.2 \%$

$-$

$0.2 \%$

-- $\quad 0.2 \mathrm{mN} / \mathrm{m}$
$7 / 90-3 / 91$

$7 / 90-3 / 91$

$7 / 90-3 / 91$

in prog

bis-difluoromethylether

Gillis; NIST; USA

sound speed

Schmidt; NIST; USA

critical parameters

density

refractive index

surface tension

$\begin{array}{ccc}- & 0.01 \% & .998 \\ & & \\ - & 0.01 \mathrm{~g} / \mathrm{cm}^{3} & - \\ 41 & - & - \\ 41 & 0.001 & - \\ 74 & 0.15 \mathrm{mN} / \mathrm{m} & -\end{array}$

in prog

in prog

in prog

in prog

in prog

2-, 3-, and 4-carbon fluoro- and hydrofluoroethers

Adcock; U. Tennessee; USA

vapor pressure

saturation density

pressure-volume-temperature

critical parameters

heat capacity

viscosity

$\begin{array}{ccccc}\mathrm{l}-\mathrm{v} & \mathrm{T}_{\text {nbp }}-\mathrm{T}_{\mathrm{c}} & - & -- & 2 \% \\ \mathrm{sl} & \mathrm{T}_{\text {nbp- }} \mathrm{T}_{\mathrm{c}} & - & -- & 2 \% \\ -- & -- & -- & 6 & 2 \% \\ -- & - & - & -- & -- \\ -- & \mathrm{T}_{\text {nbp }} \mathrm{T}_{\mathrm{c}} & - & -- & 2 \% \\ \mathrm{sl} & \mathrm{T}_{\text {nbp- }} \mathrm{T}_{\mathrm{c}} & - & -- & 2 \%\end{array}$

.9998

.9998

.9998

.9998

.9998

.9998
$8 / 88-2 / 92$ [Ad90]

$8 / 88-2 / 92$ [Ad90]

$8 / 88-2 / 92$ [Ad90]

$8 / 88-2 / 92$ [Ad90]

$8 / 88-2 / 92$ [Ad90]

8/88-2/92 [Ad90]

3- and 4-carbon hydrofluorocarbons and hydrochlorofluorocarbons

DesMarteau; Clemson U.; USA

vapor pressure

saturation density

pressure-volume-temperature

critical parameters

heat capacity

viscosity

$\begin{array}{ccccc}\text { l-v } & \mathrm{T}_{\text {nbp- }} \mathrm{T}_{\mathrm{c}} & - & -- & 2 \% \\ \mathrm{sl} & \mathrm{T}_{\mathrm{nbp}}-\mathrm{T}_{\mathrm{c}} & - & -- & 2 \% \\ -- & -- & -- & 6 & 2 \% \\ -- & - & - & -- & -- \\ -- & \mathrm{T}_{\text {nbp- }}-\mathrm{T}_{\mathrm{c}} & - & -- & 2 \% \\ \mathrm{sl} & \mathrm{T}_{\text {nbp }}-\mathrm{T}_{\mathrm{c}} & - & -- & 2 \%\end{array}$

$\begin{array}{lll}.98+ & 8 / 88-8 / 91 & {[\mathrm{Hw} 90]} \\ .98+ & 8 / 88-8 / 91 & {[\mathrm{Hw} 90]} \\ .98+ & 8 / 88-8 / 91 & {[\mathrm{Hw} 90]} \\ .98+ & 8 / 88-8 / 91 & {[\mathrm{Hw} 90]} \\ .98+ & 8 / 88-8 / 91 & {[\mathrm{H} w 90]} \\ .98+ & 8 / 88-8 / 91 & {[\mathrm{Hw} 90]}\end{array}$




\section{Other Pure Fluids (continued)}

Notes and References

[Ad90] J. L. Adcock, New chemical alternatives to the chlorofluorocarbons, paper presented at AIChE Spring National Meeting, March, 1990, Orlando, Florida.

[Hw90] S. H. Hwang, A. Beyerlein, and D. D. DesMarteau, Measurement of the heat capacity of CFCs by differential scanning calorimetry, paper presented at AIChE Spring National Meeting, March, 1990, Orlando, Florida.

[Mr90] J. Morgenstern, I. Ebinger, J. Senst, and Vollmer, Thermodynamische Bewertung alternativer Kältemittel im Zusammenhang mit den TCKW-Restrikhonen, Luft und Kältetechnik $2641-44$ (1990). 
Mixture Studies

Mixture/Investigator/

/Property

R 14/23

Maurer; U. Kaiserlautern; FRG equation of state

$\mathrm{R} 23 / 13$

Maurer; U. Kaiserlautern; FRG equation of state

$\mathrm{R} 23 / 22$

Morrison; NIST; USA bubble point pressure

$\mathrm{R} 23 / 142 \mathrm{~b}$

Morrison; NIST; USA bubble point pressure

$\mathrm{R} 32 / 134 \mathrm{a}$

Holste; Texas A\&M U.; USA vapor-liquid equilibria P-V-T-x

\section{$\mathrm{R} 32 / 152 \mathrm{a}$}

Holste; Texas A\&M U.; USA vapor-liquid equilibria P-V-T-x

$$
\text { l-v } \quad 175-\mathrm{T}_{\mathrm{c}}
$$

$1, v \quad 175-400$

$0-70$

50

$--$

$0.1 \% \quad 1 / 92-6 / 92$

$0.1 \% \quad 1 / 92-6 / 92$

R125/134a

Snellson; NRC; Canada viscosity

$0-100 \quad 50$

$2.5-3.5 \% 3 / 91-10 / 91$

R125/124

Snellson; NRC; Canada viscosity

sl 253-353

$0-100 \quad 50$

$2.5-3.5 \% 3 / 91-10 / 91$

R143a/22

Tanaka; Kobe U.; Japan viscosity

Kubota; Kobe U.; Japan vapor-liquid equilibria

$\mathrm{R} 22 / 12$

Takahashi; Tohoku U.; Japan viscosity v 298-398

0.1-6 43-79

207

$0.3 \%$ comp

[Tk89] 
Mixture Studies (continued)

Mixture/Investigator/

/Property phase $\mathrm{T}(\mathrm{K})$
$\underset{p(M P a)}{\text { range of data }} x$

no. est. accuracy start/end dates

note /ref.

\section{$\mathrm{R} 22 / 134 \mathrm{a}$}

Tanaka; Kobe U.; Japan viscosity

v 293-313

$0.1-0.1 \quad 0-100$

15

$1 \%$

comp

[Ko90]

Yoshida; Matsushita Elec.; Japan vapor-liquid equilibria

$1-v \quad 273-323$

$0.4 \mathrm{~mol} \%$

comp

$\mathrm{R} 22 / 152 \mathrm{a}$

Kruse; U. Hannover; FRG

viscosity

saturated liquid density

Mayinger; T. U. München; FRG viscosity

Schramm; Phys. Chem. Inst.; FRG second virial coefficient

Ström; Chalmers U. Tech.; Swe vapor-liquid equilibria

P-V-T-X

heat capacity

viscosity

Kubota; Kobe U.; Japan vapor-liquid equilibria P-V-T-x l 243-353

sl 233-353

v $\quad 300-425$

0.1-6.5 25-75

v 233-296

l-v --

l 258-323

l 253-313

$1 \quad 258-323$

I-v 263-323

v 348-423

1-v 280-380

sl 280-380

$1-\mathrm{v} \quad 280-380$

$\mathrm{sl}, \mathrm{sv} \quad 323-\mathrm{T}_{\mathrm{c}}$

v

$\mathrm{T}, \mathrm{P}, \mathrm{\rho}$

$\mathrm{l}, \mathrm{v}$

l,v 283-353

$0.5-1.4$

l-v $\quad .65-.85 \mathrm{~T}_{\mathrm{c}}$

$?-2.5 \quad 0-100$

$0.1-2$

$0.5-2$

sat-2

0.5-2

$-$

10-90

0-100

$0-100$

76

6

$--$

4

4

$--$

$--$

$-$

$+$

$\begin{array}{lll}- & -- & 114\end{array}$

$0.1-4 \quad 30-70 \quad 26$

$10-90 \quad 66$

$10-90 \quad 66$

66

210

$\begin{array}{cc}0.3 \mathrm{~mol} \% & \text { comp } \\ 0.15 \% & \text { comp } \\ 0.5 \% & \text { in prog } \\ 2 \% & \text { comp }\end{array}$

$1.7 \%$

$0.4 \%$

comp

comp

\section{$20 \mathrm{kPa}$}

$0.3 \%$

$0.1 \%$

comp

comp

comp

[Mz90c]

[Mz90c]

[Mz90c]

$0.5 \% \quad$ comp

Canren; Tianjin U.; PRC equation of state

Bier; U. Karlsrühe; FRG critical parameters

Oellrich; U. Karlsrühe; FRG equation of state

Sami; U. Moncton; Canada heat capacity

LaRue; CRIQ; Canada bubble point pressure

$\mathrm{R} 22 / 152 \mathrm{a} / 142 \mathrm{~b}$

Maezawa; Keio U.; Japan bubble point pressure saturated liquid density vapor-liquid equilibria

l-v $280-390$

sl 280-390

l-v $\quad 280-390$

(6 comp) 71

$20 \mathrm{kPa}$

comp

$0.2 \%$ comp

[Mz90]

$0.2 \%$

comp

[Mz90]

[Mz90]

\section{$\mathrm{R} 22 / 152 \mathrm{a} / 114$}

Sami; U. Moncton; Canada heat capacity 
Mixture Studies (continued)

Mixture/Investigator/

/Property phase $\mathrm{T}(\mathrm{K})$ range of data p (MPa) no.

points est

st. start/end dates note

/ref.

$\mathrm{R} 22 / 142 \mathrm{~b}$

Kruse; U. Hannover; FRG

$$
\text { viscosity }
$$

LeNeindre; U. Paris Nord; France vapor pressure

P-V-T-x

critical parameters

sound speed

thermal conductivity

Mayinger; T. U. München; FRG viscosity

Schramm; Phys. Chem. Inst.; FRG second virial coefficient

Stephan/Taxis; U. Stuttgart; FRG thermal conductivity

Ström; Chalmers U. Tech.; Swe vapor-liquid equilibria P-V-T-X

heat capacity

viscosity

Tanaka; Kobe U.; Japan viscosity

Kubota; Kobe U.; Japan vapor-liquid equilibria

Yoshida; Matsushita Elec.; Japan vapor-liquid equilibria

Maezawa; Keio U.; Japan bubble point pressure saturated liquid density vapor-liquid equilibria

Kumagai; Keio U.; Japan vapor-liquid equilibria P-V-T-x

\begin{tabular}{|c|c|c|c|c|c|c|c|}
\hline 1 & $237-353$ & $?-2.5$ & $0-100$ & 72 & $4 \%$ & comp & \\
\hline $1-v$ & $298-?$ & - & $40 / 60$ & -- & -- & $10 / 90-->$ & \\
\hline $\mathrm{l}, \mathrm{v}$ & $298-413$ & $0.1-100$ & $40 / 60$ & -- & -- & $10 / 90-->$ & \\
\hline - & - & - & $40 / 60$ & -- & -- & $10 / 90->$ & \\
\hline $1, v$ & $298-523$ & $0.1-100$ & $40 / 60$ & -- & -- & $10 / 90->$ & \\
\hline $1, v$ & $298-523$ & $0.1-100$ & $40 / 60$ & -- & -- & 10/90--> & \\
\hline $\mathrm{v}$ & $300-425$ & $0.1-6.5$ & $25-75$ & -- & $0.5 \%$ & $9 / 90-12 / 90$ & \\
\hline $\mathrm{v}$ & $248-296$ & - & -- & 4 & $1 \%$ & comp & \\
\hline $\mathrm{v}$ & $273-423$ & $?-5$ & -- & 100 & $1 \%$ & $-->9 / 91$ & \\
\hline l-v & -- & $0.1-2$ & -- & -- & $0.3 \mathrm{~mol} \%$ & comp & \\
\hline l & $258-323$ & $0.5-2$ & -- & -- & $0.15 \%$ & comp & \\
\hline 1 & $253-313$ & sat-2 & -- & -- & $0.5 \%$ & in prog & \\
\hline 1 & $258-323$ & $0.5-2$ & -- & -- & $2 \%$ & $\operatorname{comp}$ & \\
\hline $\mathrm{v}$ & $298-323$ & $0.1-0.1$ & $0-100$ & 11 & $1 \%$ & comp & [Ko89] \\
\hline $1-v$ & $263-338$ & - & -- & 174 & $1.5 \%$ & comp & \\
\hline $1-v$ & $273-323$ & - & - & -- & $0.4 \mathrm{~mol} \%$ & comp & \\
\hline $1-v$ & $280-400$ & - & $20-80$ & 46 & $20 \mathrm{kPa}$ & comp & [Mz90b] \\
\hline sl & $280-400$ & - & $20-80$ & 46 & $0.2 \%$ & comp & [Mz90b] \\
\hline I-v & $280-400$ & - & $20-80$ & 46 & $0.1 \%$ & comp & [Mz90b] \\
\hline $1-\mathrm{v}$ & $321-T_{c}$ & - & $20-80$ & 31 & $0.1 \%$ & comp & [Km90] \\
\hline $1, \mathrm{v}$ & $297-443$ & $0.5-10$ & $20-80$ & 445 & $0.1 \%$ & comp & [Km90] \\
\hline $1-v$ & $243-373$ & - & $0-100$ & -- & $0.5 \mathrm{~mol} \%$ & $5 / 90-9 / 90$ & \\
\hline $\mathrm{v}$ & - & -- & -- & - & - & -- & \\
\hline$T, P, \rho$ & - & - & $0-100$ & - & $0.05 \mathrm{~K} ; 6 \mathrm{kPa}$ & a comp & [Bi90] \\
\hline $1, \mathrm{v}$ & - & - & - & - & - & ?/89-?/92 & \\
\hline $1-v$ & $.65-.85 \mathrm{~T}_{\mathrm{c}}$ & - & $0-100$ & - & $1 \%$ & comp & [Lr90] \\
\hline$l-v$ & $268-383$ & - & 50 & 10 & - & comp & \\
\hline
\end{tabular}
vapor-liquid equilibria

Canren; Tianjin U.; PRC equation of state

Bier; U. Karlsrühe; FRG critical parameters

Oellrich; U. Karlsrühe; FRG equation of state

LaRue; CRIQ; Canada bubble point pressure

Morrison; NIST; USA bubble point pressure 
Mixture/Investigator/

/Property

\section{$\mathrm{R} 22 / 152 \mathrm{a} / 124$}

Yujun; Zhejiang U.; PRC vapor-liquid equilibria phase $\mathrm{T}(\mathrm{K})$ range of data
$\mathrm{p}(\mathrm{MPa})$ no. est. accuracy start/end

dates note

/ref.

R22/114

Mayinger; T. U. München; FRG viscosity$$
1-\mathrm{V} \quad--
$$$$
\text { vapor-liquid equilibria }
$$$$
\text { P-V-T- } \mathrm{x}
$$$$
\text { heat capacity }
$$$$
\text { viscosity }
$$$$
1 \quad 258-323
$$$$
1 \quad 253-313
$$$$
1258-323
$$$$
0.1-5.3 \quad 25-75
$$$$
\text { 0.1-2 -- }
$$$$
\text { 0.5-2 -- }
$$$$
\text { sat-2 -- }
$$$$
\text { 0.5-2 -- }
$$$$
\mathrm{sl}, \mathrm{sv} \quad 230-\mathrm{T}_{\mathrm{c}}
$$

V-L-E correlation critical region correlation

Kubota; Kobe U.; Japan vapor-liquid equilibria

Gorenflo; U. Paderborn; FRG vapor pressure sat. liquid density sat. vapor density critical parameters

Sami; U. Moncton; Canada heat capacity

LaRue; CRIQ; Canada bubble point pressure

\section{R22/123}

Schramm; Phys. Chem. Inst.; FRG second virial coefficient

v 268-296

$0.5 \mathrm{~mol} \% \quad 2 / 91-7 / 91$

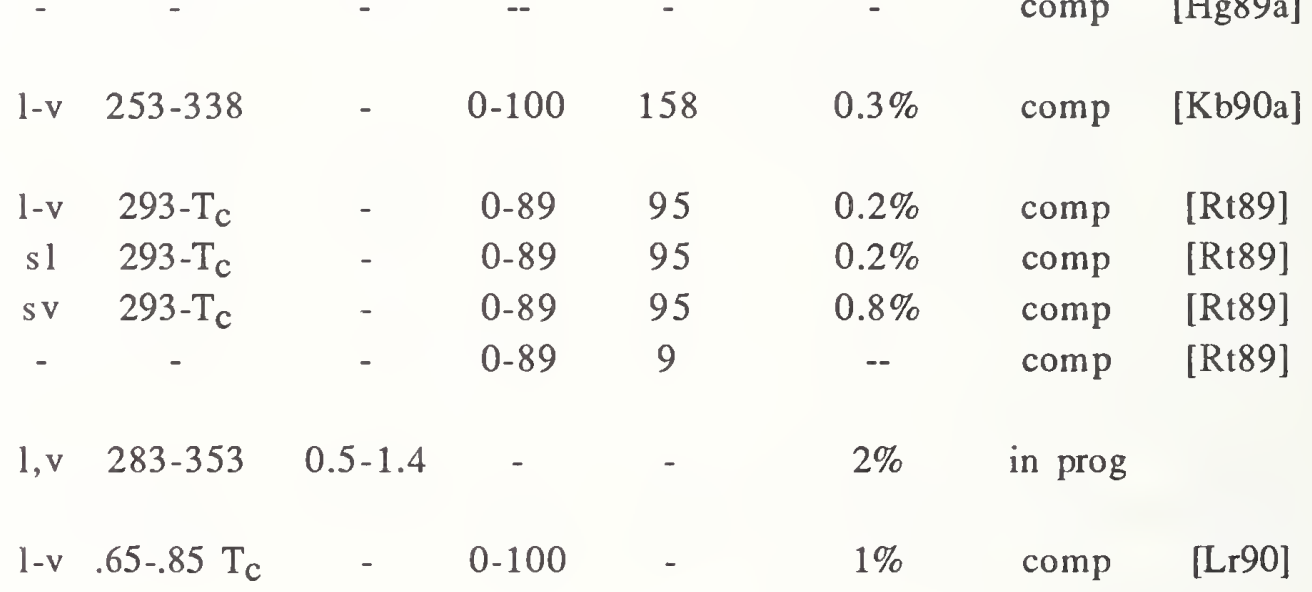

$\mathrm{R} 134 \mathrm{a} / 152 \mathrm{a}$

Baehr; U. Hannover; FRG

$$
\text { vapor-liquid equilibria }
$$
P-V-T-x

Tamatsu; Keio U.; Japan vapor-liquid equilibria P-V-T-X

Holste; Texas A\&M U.; USA vapor-liquid equilibria P-V-T-x

Bier; U. Karlsrühe; FRG vapor pressure critical parameters

Oellrich; U. Karlsrühe; FRG equation of state

Morrison; NIST; USA bubble point pressure $1-\mathrm{v} \quad 300-373$

- 25-75 40

l,v 293-433

$0.1-16 \quad 25-75 \quad 500$

l-v $300-T_{c}$

$1, \mathrm{v} \quad 300-443$

$\begin{array}{ccc}- & 20-80 & -- \\ 0.5-10 & 20-80 & --\end{array}$

l-v $180-\mathrm{T}_{\mathrm{c}}$

l,v $\quad 180-400$

$-$

1-v 203-T

$0-100$

l,v

$1-\mathrm{v} \quad 268-\mathrm{T}_{\mathrm{c}}$

$\begin{array}{cc}0.3 \mathrm{~mol} \% & \text { comp } \\ 0.15 \% & \text { comp } \\ 0.5 \% & \text { in prog } \\ 2 \% & \text { comp }\end{array}$

-- comp

[Hg89]

comp

[Hg89a]

[Lr90]

comp

$3 \%$ comp


Mixture Studies (continued)

Mixture/Investigator/

/Property

$\mathrm{R} 134 \mathrm{a} / 134$

Morrison; NIST; USA

bubble point pressure

$\mathrm{R} 134 \mathrm{a} / 123$

Kubota; Kobe U.; Japan vapor-liquid equilibria

Nakaiwa; Nat. Chem. Lab; Japan equation of state

\section{R134a/141b}

Kubota; Kobe U.; Japan vapor-liquid equilibria

R152a/134

Maezawa; Keio U.; Japan bubble point pressure saturated liquid density vapor-liquid equilibria

Morrison; NIST; USA bubble point pressure

$\mathrm{R} 152 \mathrm{a} / 142 \mathrm{~b}$

Maezawa; Keio U.; Japan bubble point pressure saturated liquid density vapor-liquid equilibria

\section{R152a/114}

Higashi; Iwaki Meisei U.; Japan V-L-E correlation

\section{R227/123}

Gorenflo; U. Paderborn; FRG bubble-point pressure saturated liquid density saturated vapor density phase $\mathrm{T}(\mathrm{K})$

l-v $268-\mathrm{T}_{\mathrm{c}}$ range of data

no.

est.

start/end

note $\mathrm{p}(\mathrm{MPa})$

points

accuracy

dates

/ref.

l-v 288-333

-- 313-363

$0.2-3.2$

10

comp

$$
\text { l-v 278-333 }
$$

$0.1 \%$

comp

[Kb90]

$\begin{array}{cccccccc}\text { l-v } & 280-380 & - & 20-80 & 44 & 20 \mathrm{kPa} & \text { comp } & \text { [Mz91] } \\ \text { sl } & 280-380 & - & 20-80 & 44 & 0.2 \% & \text { comp } & \text { [Mz91] } \\ \text { l-v } & 280-380 & - & 20-80 & 44 & 0.1 \% & \text { comp } & \text { [Mz91] } \\ & & & & & & & \\ \text { l-v } & 268-383 & - & 50 & 10 & - & \text { comp } & \end{array}$

$\begin{array}{cccccccc}\text { l-v } & 280-400 & - & 20-80 & 48 & 20 \mathrm{kPa} & \text { comp } & \text { [Mz90a] } \\ \text { sl } & 280-400 & - & 20-80 & 48 & 0.2 \% & \text { comp } & \text { [Mz90a] } \\ \text { l-v } & 280-400 & - & 20-80 & 48 & 0.1 \% & \text { comp } & \text { [Mz90a] }\end{array}$

$\mathrm{sl}, \mathrm{sv} \quad 230-\mathrm{T}_{\mathrm{c}}$

comp

[Hg89]

$\begin{array}{ccccccc}\text { l-v } & 293-423 & - & (3 \mathrm{comp}) & -- & -- & 9 / 90--> \\ \text { sl } & 293-423 & - & (3 \mathrm{comp}) & -- & -- & 9 / 90--> \\ \text { sv } & 293-423 & - & (3 \mathrm{comp}) & -- & -- & 9 / 90-->\end{array}$


Notes and References

[Bi90] K. Bier, R. Hamberger, M. Weisenburger, and J. Zhai; DKV-Jahrestagung 22/23 (1990).

[Hg89] Y. Higashi, Proc., 2nd. Asian Thermophysical Properties Conf., pp 525-530 (1989).

[Hg89a] Y. Higashi, JSME International Journal, Series II $31573-580$ (1989).

[Kb90] H. Kubota, J. Chem. Eng. Data, in press (1990).

[Kb90a] H. Kubota, J. Chem. Eng. Japan 23155 (1990).

[Km90] K. Kumagai, submitted to J. Chem. Eng. Data (1990).

[Ko89] thesis, Kobe University (1989).

[Ko90] thesis, Kobe University (1990).

[Lr90] P. Larue, P. Renaud, and M. Parent, Centre de recherche industrielle du Québec technical report no. FAB-90-007, May, 1990.

[Mz90] Y. Maezawa, to be presented at 31st High Pressure Conference of Japan, November, 1990, Osaka.

[Mz90a] Y. Maezawa, to be presented at 11th Japan Symposium on Thermophysical Properties, November, 1990 , Tokyo.

[Mz90b] Y. Maezawa, presented at 12th European Conference on Thermophysical Properties, September, 1990, Vienna.

[Mz90c] Y. Maezawa, to be published in Fluid Phase Equilibria (1990).

[Mz91] Y. Maezawa, to be presented at 3rd ASME/JSME Thermal Engineering Joint Conference, March, 1991, Reno, Nevada.

[Nb90] H. Nabizadeh and F. Mayinger, Viscosity of refrigerants R22, R114 and mixtures of R22+R114 at superheated and supercritical states, paper presented at AIChE Spring National Meeting, March, 1990, Orlando, Florida.

[Nk90] M. Nakaiwa, et al., Proc. 3rd IEA Heat Pump Conference, pp 693-701 (1990).

[P190] B. Platzer, A. W. Polt, and G. Maurer, Thermophysical Properties of Refrigerants, Springer-Verlag, Heidelberg (1990).

[Rt89] W. Rott, dissertation, Universität -GH- Paderborn (1989).

[Tk89] M. Takahashi, Trans. of the JAR 6255 (1989). 
RESPONDING ORGANIZATIONS 
Australian National University

Research School of Physical Sciences

Canberra, A.C.T. 26

principal investigator:

L. A. Woolf

facsimile:

other researcher:

R. K. Malhotra

apparatus and techniques employed:

pressure-volume-temperature:

viscosity:

sample purity analysis:

Centre de Recherche Industrielle du Québec

333 rue Franquet

Case Postale 9038

Sainte-Foy, Québec G1V 4C7

principal investigator

Mr. Michel Boulanger

Paul Larue

facsimile

apparatus and techniques employed:

vapor-liquid equilibria

University of Moncton

Department of Mechanical Engineering

Moncton, New Brunswick E1A 3E9

principal investigator:

Prof. Samuel Sami

facsimile

other researchers:

W. Leblanc

L. Fang

J. Schnotale
61-6-249-2400

61-6-249-1884

bellows volumometer; vibrating tube densimeter not specified

supplier's analysis

\section{CANADA}

1-418-659-1550

$1-418-659-1550$

$1-418-652-2251$

variable volume cell

1-506-858-4194

$1-506-858-4585$

1-506-858-6916

$1-506-858-4871$

$1-506-858-4871$ 
CANADA (continued)

National Research Council of Canada

Low Temperature Laboratory

Montreal Road

Ottawa, Ontario K1A 0R6

principal investigators:

W. Keith Snelson

Jeff Linton

facsimile

apparatus and techniques employed: viscosity

University of New Brunswick

P.O. Box 4400

Fredericton, New Brunswick E3B 5A3

principal investigator:

Prof. James Venart

facsimile

other researchers:

R. C. Prasad

G. H. Wang

apparatus and techniques employed:

thermal conductivity/diffusivity
$1-613-993-4892$

$1-613-954-1235$

falling ball viscometer

$1-506-453-4509$

1-506-453-5025

transient hot wire 
Tianjin University

Thermal Energy Research Institute

Tianjin 300072

principal investigator:

Prof. Lu Canren

facsimile:

other researchers:

Dr. Ma Yitai

Prof. He Zhimai

techniques employed:

equation of state:

Tsinghua University

Department of Thermal Engineering

Beijing, 100084

principal investigator:

Zhu Mingshan

facsimile:

other researchers:

Wang Bu-Xuan

Lin Zhao-Zhuang

Han Li-Zhong

apparatus and techniques employed:

vapor pressure:

saturation density:

pressure-volume-temperature:

sound speed:

surface tension:

thermal conductivity:

viscosity:

sample purity analysis:
86-33-3704-3451

86-33-318329

$86-33-3704-2821$

Redlich-Kwong-Soave and Martin-Hou equations

86-1-2561141 x2987

$86-1-2562768$

constant volume method

magnetic suspension densimeter

Burnett method

accoustic method

capillary rise method

steady-state method

capillary method

supplier's analysis 
Xi'an Jiaotong University

Department of Power Machinery Engineering

Xi'an, Shaanxi Province 710049

principal investigator:
J. M. Yin
$\mathrm{cc}+32911-727$
facsimile:
cc+029-335471

other researchers:

J. X. Guo

L. C. Tan

Z. Y. Zhao

apparatus and techniques employed:

vapor pressure:

saturation density:

critical parameters:

sample purity analysis:

constant volume method

not specified

observation of meniscus for temperature and density

not specified

Zhejiang University

Laboratory of Chemical Engineering Thermodynamics

Department of Chemical Engineering

Hangzhou, 310027

principal investigator:

Prof. Hou Yujun

telex:

cc+572244-2921

35040 ZUFAO CN

other researchers:

Prof. Wu Zhaoli

$\mathrm{cc}+572244-3246$

Dr. Chen Guangming

Li Changsen

cc+572244-2621

cc $+572244-2621$

apparatus and techniques employed:

mixture V-L-E:

Rose-William cell; vapor circulation technique;Martin-Hou

sample purity analysis:

equation of state

own analysis 
Czech Technical University

College of Mechanical Engineering

Technikká 4

16607 Prauge 6

principal investigator:

Dr. Václav Vacek

$\mathrm{cc}+332-2581$

facsimile

$\mathrm{cc}+311-2768$

other researchers:

Jiri Petrak

apparatus and techniques employed pressure-volume-temperature: sample purity analysis:

constant volume piesometer own analysis

\section{FRANCE}

Universite Paris Nord

Laboratoiré d'Ingénierie des Materiaux et Hautes Pressions

Avenue J. B. Clément

93430 Villetaneuse

principal investigator:

Dr. B. LeNeindre

$33-1-49-40-34-38$

facsimile

$33-1-49-40-33-33$

other researchers:

apparatus and techniques employed

vapor pressure:

not specified

pressure-volume-temperature:

vibrating tube densimeter

critical parameters:

sound speed:

thermal conductivity:

sample purity analysis:

not specified

not specified

coaxial cylinder

supplier's analysis 
Universität Duisburg

Fachgebiet Thermodynamik

Lotharstraße 1

4100 Duisburg

principal investigator:

Prof. Dr.-Ing. Klaus Lucas

other researchers:

U. Delfs

apparatus and techniques employed ideal gas properties:

Universität Erlangen-Nürnberg

Lehrstuhl Für Technische Thermodynamik

Am Weichselgarten 9

8520 Erlangen

principal investigator:

Prof. Dr.-Ing. A. Leipertz

facsimile

apparatus and techniques employed

sound speed:

thermal diffusivity:

sample purity analysis:
49-2031-379-3355

statistical thermodynamics
49-9131-699-162

49-9131-699-161

photon correlation spectroscopy photon correlation spectroscopy not specified

Universität Hannover

Institut für Kältetechnik und Angewandte Wärmetechnik

Welfengarten 1a

D-3000 Hannover 1

principal investigator:

Prof. Dr.-Ing. H. Kruse

49-511-762-2358

facsimile

49-511-762-5203

apparatus and techniques employed

vapor pressure:

saturated liquid density:

viscosity:

sample purity analysis:

predictive methods:

not specified

pycnometer

falling ball viscometer

own analysis

functional group methods 
Universität Hannover

Institut für Thermodynamik

Callinstraße 36

D-3000 Hannover 1

principal investigator:

Prof. Dr.-Ing. H. D. Baehr

facsimile

apparatus and techniques employed

vapor pressure:

saturated liquid density:

pressure-volume-temperature:

mixture V-L-E

sample purity analysis:

Universität Gesamthochschule Paderborn

Laboratorium für Wärme- und Kältetechnik

Pohlweg 55

D-4790 Paderborn

principal investigator:

Prof. Dr.-Ing D. Gorenflo

facsimile

other researchers:
W. Rott
H. Schömann
S. Caplanis
G. Herres

apparatus and techniques employed

vapor pressure:

saturation density:

sample purity analysis:

Universität Kaiserslautern

Lehrstuhl für Technische Thermodynamik

Erwin-Schrödinger-Straße

Postfach 3049

D-6750 Kaiserslautern

principal investigator:

Prof. Dr.-Ing. G. Maurer

facsimile

other researchers:

Dipl.-Ing. B. Platzer

apparatus and techniques employed

equation of state:
49-631-205-2311

49-631-205-3055

49-511-762-2277

49-511-762-3456

not specified

vibrating tube densimeter

Burnett apparatus; vibrating tube densimeter

VLE apparatus

own analysis

49-5251-60-2393

$49-5251-60-3207$ or -2519

$49-5251-60-2391$

$49-5251-60-2391$

$49-5251-60-2390$

49-5251-60-2394

static equilibrium cell

static equilibrium cell with vibrating tube densimeter own analysis

49-631-205-2410

Bender equation of state 
Universität Karlsrühe

Institute für Physikalische Chemie und Elektrochemie

Kaiserstraße 12, Postfach 6380

7500 Karlsruhe 1

principal investigator:

Prof. Dr. E. U. Franck

49-721-608-2101

facsimile

49-721-696-324

apparatus and techniques employed dielectric constant:

capacitance technique

Universität Karlsrühe

Institute für Technische Thermodynamik und Kältetechnik

Postfach 6980

7500 Karlsruhe 1

principal investigator:

Prof. Dr. K. Bier

Prof. Dr.-Ing. L. Oellrich

facsimile

apparatus and techniques employed

vapor pressure:

pressure-volume-temperature:

critical parameters:

heat capacity:

sample purity analysis:

equation of state:

Technische Universität München

Lehrstuhl A für Thermodynamik

Archisstraße 21

8000 München 2

principal investigators:

Prof. Dr.-Ing. J. Straub

Prof. Dr.-Ing. F. Mayinger

facsimile

other researchers:

Dipl.-Ing. B. Kruppa

Dr.-Ing. H. Nabizadeh

apparatus and techniques employed

pressure-volume-temperature:

critical parameters:

surface tension:

thermal diffusivity:

refractive index:

viscosity:

sample purity analysis:
49-721-608-2321

49-721-608-2332

49-721-66-1874

static equilibrium cell

Burnett apparatus

static equilibrium cell

adiabatic flow calorimeter

own analysis \& supplier's analysis

not specified

49-89-2105-3438

49-89-2105-3435

49-89-2105-3451

49-89-2105-3452

49-89-2105-3436

not specified

not specified

capillary rise

dynamic light scattering

not specified

oscillating disk viscometer

supplier's analysis 
Physikalisch-Chemisches Institut der Universität

Im Neuenheimer Feld 253

D-6900 Heidelberg

principal investigator:

Prof. B. Schramm

apparatus and techniques employed

pressure-volume-temperature: sample purity analysis:

Physikalisch-Technische Bundesanstalt

Heat Division

Bundesallee 100

D-3300 Braunschweig

principal investigators:

Dr. W. Blanke

Dr. W. Hemminger

facsimile

other researchers:

Dr. R. Weiß

Dr. U. Hammerschmidt

apparatus and techniques employed

vapor pressure:

pressure-volume-temperature:

triple point temperature:

thermal conductivity:

sample purity analysis:

Universität Rostock

Sektion Chemie

WB Physikalische Chemie

Hermannstraße 14

Rostock 1, O-2500

principal investigators:

Dr. J. K. Lehmann

Doz. Dr. J. Millat

facsimile

apparatus and techniques employed dielectric constant:

sample purity analysis:
$49-6221-563456$

measurement of second virial coefficients

supplier's analysis

49-531-592-3110

49-531-592-3211

constant volume method constant volume method not specified guarded hot plate supplier's analysis

37-81-25509

37-81-25509

37-81-369-666

cyclic expansion method own analysis 
Ruhr Universität Bochum

Institüt für Thermodynamik

Postfach 102148

D-4630 Bochum 1

principal investigator:

Prof. Dr.-Ing. W. Wagner

facsimile

other researchers:

apparatus and techniques employed vapor pressure:

saturated liquid density:

saturated vapor density:

pressure-volume-temperature:

sample purity analysis:

equation of state:

Ruhr Universität Bochum

Institüt für Thermo- und Fluiddynamik

P.O. Box 102148

D-4630 Bochum 1

principal investigator:

Prof. F. Kohler

facsimile

other researchers:

R. Kohlen

apparatus and techniques employed vapor pressure:

pressure-volume-temperature:

sound speed

sample purity analysis:
49-234-700-3030

49-234-700-2541

single- and dual-sinker densimeters single- and dual-sinker densimeters single- and dual-sinker densimeters single- and dual-sinker densimeters supplier's analysis

multi-property fit

49-234-700-6389

49-234-700-2541

isochoric PVT

isochoric PVT; Burnett apparatus

spherical resonator

own analysis 
Ruhr Universität Bochum

Thermo- und Fluiddynamik

Wärme und Stoffübertragung

Universitätstraße 150

D-4630 Bochum 1

principal investigator:
M. Fiebig
49-234-700-3083
facsimile
49-234-700-2000

other researchers:
M. Ibreighith
49-234-700-6680
G. Wu
49-234-700-6680
J. Wang
49-234-700-6680

apparatus and techniques employed

thermal diffusivity:

sample purity analysis:

photon correlation spectroscopy

supplier's analysis

Universität Stuttgart

Institut für Techniche Thermodynamik und Thermische Verfahrenstechnik

Pfaffenwaldring 9

D-7000 Stuttgart 80

principal investigator:

Prof. Dr.-Ing. K. Stephan 49-711-685-6102

facsimile

$49-711-685-6140$

other researchers:

R. Krauss

Dipl.-Ing. B. Taxis

$49-711-685-6566$

49-711-685-6113

apparatus and techniques employed

thermal conductivity:

sample purity analysis:

data base project

transent hot wire

supplier's analysis

Universität Stuttgart

Institut für Thermodynamik und Wärmetechnik

Pfaffenwaldring 6

7000 Stuttgart 80

principal investigators:

Dr. U. Gross

49-711-685-3536

Prof. E. Hahne

49-711-685-3536

facsimile

49-711-685-3280

apparatus and techniques employed

thermal conductivity:

transient hot wire

sample purity analysis:

not specified 
Hochschule für Verkehrswesen

Friedrich-List-Platz 1

Dresden, O-8010

principal investigator:

Dipl.-Ing. J. Morgenstern

other researchers:

Dr. I. Ebinger

Dr. J. Senst

apparatus and techniques employed equation of state:
$37-51-462-2592$

Redlich-Kwong-Soave equation; incremental methods (Lydersen, Riedel, Westmeier, etc.) 
Aristotle University

Chemical Engineering Department

University Box 453

GR54001 Thessaloniki

principal investigator:

Prof. Marc J. Assael

facsimile:

other researchers:

Mr. L. Karagiannidis

apparatus and techniques employed: thermal conductivity:

viscosity:

sample purity analysis:
30-31-991532

30-31-206138

transient hot wire

vibrating wire

not specified

\section{INDIA}

91-812-344411 x2249

91-812-341683

corresponding states 
Universita di Ancona

Dipartimento di Energetica

Via Brecce Bianche

I-60100 Ancona

principal investigator:

Carlo Baroncini

facsimile

other researchers:

Marco Pacetti

Fabio Polonara

Giuliani Giuliano

apparatus and techniques employed vapor pressure:

pressure-volume-temperature: sample purity analysis:
39-71-5893765

39-71-894677

$39-71-894780$

39-71-5893765

39-71-5893766

constant volume technique constant volume technique own analysis 
Asahi Glass Company, Ltd.

10, Goikaigan, Ichihara-shi, Chiba 290

principal investigator:

M. Fukushima

facsimile:

other researchers:

N. Watanabe

T. Kaminura

apparatus and techniques employed:

vapor pressure:

saturation density:

pressure-volume-temperature:

critical parameters:

sample purity analysis:

Iwaki Meisei University

5-5-1 Iino, Chuodai, Iwaki 970

principal investigator:

Y. Higashi

facsimile

apparatus and techniques employed surface tension:

mixture V-L-E:

Kanagawa Institute of Technology

Shimo-ogino 1030, Atsugi, 243-02

principal investigator:

Dr. K. Oguchi

facsimile:

other researchers:

Y. Takaishi

N. Yada

apparatus and techniques employed vapor pressure:

saturation density:

pressure-volume-temperature

equation of state:
81-436-23-3151

$81-436-23-3164$

constant volume method

buoy method; constant volume method

constant volume method

visual observation of meniscus for density and temperature own analysis

$81-246-29-5111$

$81-246-28-5415$

Wilhelmy method

original correlation

$81-462-41-1211$

$81-462-42-3737$

constant volume method

buoy method; constant volume method

constant volume method

extended Bennedict-Webb-Rubin 
Keio University

Thermodynamics Laboratory

Faculty of Science and Technology

3-14-1, Hiyoshi, Kohoku-ku, Yokohama 223

principal investigators:

\section{Prof. Koichi Watanabe}

Dr. Haruki Sato

facsimile

graduate students and other collaborators:
N. Ehara
Y. Hayakawa

T. Hori

A. Hoshi

K. Kumagai

Y. Maezawa

H. Miyahara

T. Naito

A. Ogino

S. Okazaki

Z. Y. Qian

A. Saitoh

N. Sezaki

S. Tanakawa

T. Shimokawa

J. Tatoh

apparatus and techniques employed vapor pressure:

saturated liquiã density:

saturated vapor density:

pressure-volume-temperature:

critical parameters:

heat capacity:

sample purity analysis:

equation of state:

Keio University

3-14-1, Hiyoshi, Kohoku-ku, Yokohama 223

principal investigators:
A. Nagashima
Y. Nagasaka
facsimile:

apparatus and techniques employed

thermal conductivity:

viscosity:

sample purity analysis:

\author{
$81-45-563-1141 \quad x 3127$ \\ $81-45-563-1141 \times 3106$ \\ $81-45-563-3421$
}

magnetic densimeter; constant volume method; Burnett method

magnetic densimeter; visual critical point cell; constant volume method

visual critical point cell

magnetic densimeter; constant volume method; Bumettisochoric method

visual cell for temperature and density

flow calorimeter

supplier's analysis

25-term virial

81-44-63-1141

81-44-63-1141

81-44-62-7625

transient hot wire

capillary viscometer

supplier's analysis 
Kobe University

Department of Chemical Engineering

Rokkodai, Nada, Kobe 657

principal investigators

Prof. T. Makita

Dr. Y. Tanaka

Dr. S. Matsuo

Dr. H. Kubota

facsimile:

apparatus and techniques employed vapor pressure:

pressure-volume-temperature

critical parameters:

heat capacity:

mixture V-L-E

dielectric constant:

thermal conductivity:

viscosity:

sample purity analysis:

Kyoto Institute of Technology

Department of Chemical Engineering

Faculty of Engineering and Design

Matsugasaki, Sakyoku, Kyoto 606

principal investigators

T. Takagi

J. Yata

facsimile:

other researchers:

M. Hori

T. Minamiyama

C. Kawashima

apparatus and techniques employed

sound speed:

thermal conductivity:

sample purity analysis:
81-78-881-1212

$81-78-881-1212$

$81-78-881-1212$

$81-78-881-1212$

$81-78-802-3564$

constant volume method

vibrating-tube densimeters (commercial and modified);

constant volume method; Burnett method; high-pressure

Burnett method

observation of meniscus

flow calorimeter

vapor liquid circulation method

frequency counting method; LCR meter

stationary coaxial cylinder; transient hot wire

rolling ball viscometer; falling cylinder viscometer

supplier's analysis

$81-75-791-3211$

$81-75-791-3211$

$81-75-711-9843$

sing-around method

transient hot wire

supplier's analysis 
Matsushita Electric Industrial Company, Ltd.

Living Environmental Systems Research Lab

3-15 Yagumo-Nakamachi, Moriguchi, Osaka 570

principal investigator

Yuji Yoshida

$81-6-906-4844$

facsimile:

$81-6-906-4590$

other researchers:

Koji Arita

Masami Funakura

apparatus and techniques employed

mixture V-L-E

static V-L-E method

sample purity analysis:

supplier's analysis

Nagaoka University of Technology

Department of Mechanical Engineering

1603-1, Kamitomioka, Nagaoka, Niigata 940-21

principal investigator

Masaaki Okada

facsimile

$81-258-46-6000 \quad x 7143$

$81-258-46-6972$

other collaborators:

M. Hattori

K. Watanabe

apparatus and techniques employed

surface tension:

sample purity analysis:

single and differential capillary rise

supplier's analysis

National Chemical Laboratory for Industry

Chemical Systems Division

1-1, Higashi, Tsukuba-shi 305

Ibaraki

principal investigator

T. Akiya

81-298-54-4661

facsimile:

81-298-54-4487

other researcher:

M. Nakaiwa

apparatus and techniques employed:

mixture V-L-E:

not specified

sample purity analysis:

own analysis 
Tohoku University

Chemical Research Institute of Non-Aqueous Solutions

2-1-1 Katahira, Aoba-ku, Sendai 980

principal investigators:
S. Takahashi
$81-22-227-6200 \times 3446$
C. Yokoyama
$81-22-227-6200 \quad x 3447$

other researchers:
A. Kumagai
$81-22-227-6200 \times 3448$
K. Otake
$81-22-227-6200 \times 3447$
M. Takahashi
$81-22-227-6200 \times 3448$
facsimile
$81-22-223-8956$

apparatus and techniques employed vapor pressure:

pressure-volume-temperature:

saturated liquid density:

viscosity:

sample purity analysis:

static method

isochoric method

pycnometer

oscillating-disk viscometer; capillary viscometer

supplier's analysis 
Ajou University

Department of Mechanical Engineering

5 Won Chon Dong

Suwon 440-380

principal investigator:

Youngmoo Park

facsimile:

techniques employed:

equation of state:

Korea Standards Research Institute

Temperature Laboratory

P.O. Box 3

Taedok Science Town

Taejou

principal investigator:

Dr. Hee-Baik Chae

facsimile:

apparatus and techniques employed:

surface tension:

refractive index:

thermal conductivity:

sample purity analysis:

Seoul National University

Department of Mechanical Engineering Seoul 151-742

principal investigator:

Sung Tack Ro

facsimile:

apparatus and techniques employed:

thermal conductivity:

sample purity analysis:

equation of state:
$82-2-231-7121 \times 646$

82-331-33-5158

Carnahan-Starling equation
82-2-820-5199

82-2-231-6813

capillary rise technique

optical method

transient hot wire

supplier's analysis
82-2-883-7111

82-2-883-0179

transient hot wire supplier's analysis

not specified 
Universidade de Lisboa

Departamento de Química

Campo Grande, Bloco Cl

1700 Lisboa

principal investigator:

Prof. Dr. Carlos Nieto de Castro

facsimile

other researchers:

U. V. Mardolcar

J. M. N. A. Fareleira

apparatus and techniques employed pressure-volume-temperature: dielectric constant: thermal conductivity: sample purity analysis:
351-1-7583141

351-1-7599404

vibrating tube densimeter capacitance measurement concentric cylinder method; transient hot wire supplier's analysis

\section{SPAIN}

Universidad Politecnica de Canarias

Cátedra de Termodinámica Escuela

Escuela Superior de Ingenieros Industriales

Tafira Baja, 35071-Las Palmas

Canary Islands

principal investigator:

Prof. Juan Ortega Saavedra

34-28-353500

facsimile

other researchers:
Dr. Santiago Matos
Dr. Juan A. Peña
Dr. Salvador Galván

apparatus and techniques employed

vapor pressure:

saturated liquid density:

heat capacity:

refractive index:

sample purity analysis:

not specified

not specified

not specified

not specified

not specified 
Chalmers University of Technology

Department of Chemical Engineering Design

S-412 96 Göteborg

principal investigators:

Dr. Krister Ström

46-31-722946

facsimile

46-31-167194

apparatus and techniques employed

pressure-volume-temperature:

heat capacity:

mixture V-L-E

viscosity:

sample purity analysis:

vibrating tube densimeter

Setaram BT calorimeter

Jones circulation unit

Höppler viscometer

supplier's analysis

\section{U.S.S.R}

The Institute of Low Temperature and Power Engineering 270057, Odessa

ul. Petra Velikugo, 1/3

principal investigators:

V. J. Los

cc $+209-170$

A. V. Los

$\mathrm{cc}+209-112$

techniques employed:

equation of state:

virial equation 
University of Bristol

School of Chemistry

Cantock's Close

Bristol BS8 1TS

principal investigator:

Dr. C. J. Wormald

facsimile

apparatus and techniques employed

enthalpy:

sample purity analysis:

Imperial College

Department of Chemical Engineering

Prince Consort Road

London SW7 2BY

principal investigators:

Prof. J. P. M. Trusler

Prof. W. A. Wakeham

Dr. K. M. DeReuck

facsimile

other researchers:
V. Vesovic
R. Craven
S. B. Cha

apparatus and techniques employed sound speed:

thermal conductivity viscosity

sample purity analysis:

equation of state:
44-272-303-030 x4719

44-272-250-612

flow calorimeter

supplier's analysis

44-71-589-5111 x4358

44-71-225-8300

44-71-584-1170

spherical resonator

transient hot wire

vibrating wire

supplier's analysis

Wagner algorithm 
National Engineering Laboratory

Energy and Environment Centre

East Kilbride

Glasgow G75 0QU

principal investigator:

Dr. J. T. R. Watson

Dr. A. I. Johns

facsimile

other researchers:

W. Gaskill

apparatus and techniques employed pressure-volume-temperature: thermal conductivity: sample purity analysis:

Polytechnic South West

Department of Environmental Sciences

Drake Circus, Plymouth

Devon PL4 8AA

principal investigator:

Dr. G. P. Matthews

facsimile

other researchers:

D. C. Dowdell

apparatus and techniques employed viscosity:

sample purity analysis:
44-3552-20222

44-3552-20222

44-3552-63398

single sinker magnetic suspension densimeter transient hot wire

supplier's analysis
44-752-233021

44-752-232293

gas phase capillary flow viscometry

not specified 
Clemson University

Department of Chemistry

Clemson, South Carolina 29634-1905

principal investigator:

Prof. Darryl D. DesMarteau

other researchers:

Dr. Adolph L. Beyerlein

Sun Hee Hwang

apparatus and techniques employed

vapor pressure:

critical parameters:

sample purity analysis:

Cornell University

School of Chemical Engineering

377 Olin Hall

Ithaca, New York 14853-5201

principal investigator:

Dr. John A. Zollweg

facsimile

apparatus and techniques employed pressure-volume-temperature: speed of sound:

sample purity analysis:

University of Idaho

Center for Applied Thermodynamic Studies

Moscow, Idaho 83843

principal investigators:

Prof. Richard T. Jacobsen

Dr. Steven Penoncello

Dr. Steven Beyerlein

facsimile

apparatus and techniques employed

equation of state: not specified

not specified

own analysis

$1-607-255-7101$

$1-607-255-9166$

gas expansion technique pulse-echo-overlap technique supplier's analysis
$1-208-885-6479$

$1-208-885-7245$

$1-208-885-7245$

$1-208-885-6645$

fundamental EOS, MBWR, selection algorithm 
University of Maryland

Institute for Physical Science and Technology

College Park, Maryland 20742

principal investigator:

Prof. Jan V. Sengers

facsimile

$1-301-405-4805$

$1-301-314-9404$

other researchers:

S. Tang

apparatus and techniques employed equation of state:

renormalization theory for critical region

National Institute of Standards and Technology

Thermophysics Division

325 Broadway, Mail Stop 838

Boulder, Colorado 80303-3328

principal investigators:
Dr. W. M. Haynes
$1-303-497-3247$
Dr. James F. Ely
$1-303-497-5467$
Dr. Tom Bruno
$1-303-497-5158$
Mr. Dwain Diller
$1-303-497-3255$
Mr. John Howley
$1-303-497-5607$
Dr. Marcia L. Huber
$1-303-497-5252$
Dr. Joe Magee
$1-303-497-3298$
Dr. Mark McLinden
$1-303-497-3580$
Dr. Vicki Niesen
$1-303-497-5614$
Ms. Stephanie Outcalt
$1-303-497-5786$
Dr. Richard Perkins
$1-303-497-5499$
Dr. Jamal Sandarusi
$1-303-497-3437$
facsimile
$1-303-497-5224$

apparatus and techniques employed vapor pressure:

saturated liquid density:

saturated vapor density:

pressure-volume-temperature:

heat capacity:

thermal conductivity:

viscosity:

sample purity analysis:

equation of state:

isochoric apparatus; V-L-E apparatus

isochoric apparatus; V-L-E apparatus; magnetic suspension densimeter

isochoric apparatus; V-L-E apparatus

isochoric apparatus; magnetic suspension densimeter;

vibrating tube densimeter

adiabatic isochoric calorimeter

transient hot wire

torsionally oscillating quartz crystal

gas chromatography/mass spectrometry; infrared and

ultraviolet spectrometry; Karl Fisher moisture analysis

Jacobsen-Stewart BWR; Schmidt-Wagner; extended

corresponding states; Carnahan-Starling-DeSantis 
National Institute of Standards and Technology

Thermophysics Division

Building 226; Room A-105

Gaithersburg, Maryland 20899

principal investigators:
Dr. Richard F. Kayser
Dr. Michael R. Moldover
Mr. Dana Defibaugh
Mr. John Gallagher
Dr. Keith Gillis
Dr. Anthony R. H. Goodwin
Dr. Christopher W. Meyer
Dr. Graham Morrison
Dr. James Schmidt
Dr. Lloyd A. Weber
facsimile

$1-301-975-2483$

$1-301-975-2459$

$1-301-975-2471$

$1-301-975-2455$

$1-301-975-2468$

$1-301-975-2443$

$1-301-975-2473$

$1-301-975-2450$

$1-301-975-2458$

$1-301-975-2462$

$1-301-869-4020$

apparatus and techniques employed vapor pressure:

saturated liquid density:

saturated vapor density:

pressure-volume-temperature:

critical parameters:

sound speed:

surface tension:

dielectric constant:

sample purity analysis:

equation of state:

variable volume cell; Burnett-isochoric apparatus;

ebulliometer

variable volume cell; vibrating-tube densimeter; visual cell variable volume cell; Burnett-isochoric apparatus; vibrating tube densimeter

Burnett-isochoric apparatus; vibrating-tube densimeter visual cell; variable volume cell

spherical acoustic resonator

differential capillary rise technique

capacitance technique

NIST-Boulder

Carnahan-Starling-DeSantis

Oak Ridge National Laboratory

Energy Division

P.O. Box 2009

Oak Ridge, Tennessee 37831

principal investigators:

Dr. James R. Sand

$1-615-574-5819$

S. K. Fischer

facsimile

$1-615-574-9338$

apparatus and techniques employed:

equation of state:

Lee-Kessler-Plöcker 
University of Tennessee-Knoxville Department of Chemistry

Knoxville, Tennessee 37996-1600

principal investigator:

Prof. James L. Adcock

facsimile

other researchers:

Prof. W. Alexander Van Hook

Bao-Hai Wang

Shyam B. Mathur

apparatus and techniques employed vapor pressure:

saturated liquid density:

pressure-volume-temperature:

critical parameters:

sample purity analysis:

Texas A\&M University

Department of Chemical Engineering

College Station, Texas 77843-3122

principal investigators:

Prof. James C. Holste

Prof. K. R. Hall

facsimile

apparatus and techniques employed vapor pressure:

pressure-volume-temperature:

critical parameters:

sample purity analysis:

Texas A\&M University

Thermodynamics Research Center

College Station, Texas 77843-3111

principal investigators:

Prof. K. N Marsh

Prof. B. E. Gammon

facsimile

apparatus and techniques employed ideal gas properties:

fluid property database:
$1-615-974-3391$

$1-615-974-3454$

isochoric apparatus

vibrating tube densimeter

vibrating tube densimeter; isochoric apparatus

visual cell

capillary gas-liquid chromatography

isochoric apparatus

isochoric apparatus; Burnett apparatus; continuously weighed pycnometer; vibrating tube densimeter visual cell

supplier's analysis

$1-409-845-4940$
$1-409-845-2930$
$1-409-845-6446$

statistical mechanics

wide variety of compounds including refrigerants 

4. TITLE AND SUBTITLE

A Survey of Current Worldwide Research on the Thermophysical Properties of Alternative Refrigerants

5. AUTHOR(S)

M.O. McLinden, W.M. Haynes, J.T.R. Watson, and K. Watanabe **

6. PERFORMING ORGANIZATION (IF JOINT OR OTHER THAN NIST, SEE INSTRUCTIONS)

U.S. DEPARTMENT OF COMMERCE

NATIONAL INSTITUTE OF STANDARDS AND TECHNOLOGY

BOULDER, COLORADO $80303-3328$

7. CONTRACT/GRANT NUMBER

8. TYPE OF REPORT AND PERIOD COVERED

9. SPONSORING ORGANIZATION MAME AND COMPLETE ADDRESS (STREET, CITY, STATE, ZIP)

U.S. Department of Energy

Office of Buildings and Community Systems

1000 Independence Ave., SW

Washington, DC 20585

\section{SUPPLEMENTARY NOTES}

*Energy and Environment Centre, National Engineering Laboratory, Glasgow, United Kingdom **Department of Mechanical Engineering, Keio University, Yokohama, Japan

11. ABSTRACT (A 200-WORD OR LESS FACTUAL SUMMARY OF MOST SIGNIFICANT INFORMATION. IF DOCUMENT INCLUDES A SIGNIFICANT BIBLIOGRAPHY LUTERATURE SURVEY, MENTION IT HERE.)

This survey represents an exhaustive compilation of the research activities throughout the world concerned with either measurements or correlations of the thermophysical properties of alternative refrigerants. The properties covered in this study include thermodynamic, transport, phase equilibria, and other properties such as dielectric constant and refractive index. This survey has included a wide range of fluids (including R23, R32, R125, R143a, R22, R134a, R152a, R134 $\mathrm{R} 124, \mathrm{R} 142 \mathrm{~b}, \mathrm{R} 123, \mathrm{R} 123 \mathrm{a}, \mathrm{R} 141 \mathrm{~b})$ along with mixtures containing at least one of these fluids. This report presents in tabular form summary information about each research activity; this survey does not present raw data or correlating equations.

12. KEY WORDS (6 TO 12 ENTRIES; ALPHABETICAL ORDER; CAPITALIZE ONLY PROPER NAMES; AND SEPARATE KEY WORDS BY SEMICOLONS)

hydrochlorofluorocarbons; hydrofluorocarbons; refrigerants; survey; thermodynamic properties; thermophysical properties: transport properties

\section{AVAILABILITY}

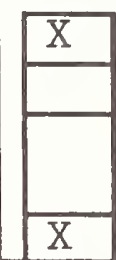

UNLIMITED

FOR OFFICIAL DISTRIBUTION. DO NOT RELEASE TO NATIONAL TECHNICAL INFORMATION SERVICE (NTIS).

ORDER FROM SUPERINTENDENT OF DOCUMENTS, U.S. GOVERNMENT PRINTING OFFICE, WASHINGTON, DC 20402.

ORDER FROM NATIONAL TECHNICAL INFORMATION SERVICE (NTIS), SPRINGFIELD, VA 22161.
14. NUMBER OF PRINTED PAGES

$$
80
$$

15. PRICE 

